\title{
Evaluation of In Vitro Models for Assessment of Human Intestinal Metabolism in Drug Discovery
}

\author{
Mari Davies, Prabha Peramuhendige, Lloyd King, Melanie Golding, Apoorva Kotian, Mark Penney, \\ Syeda Shah, and (1) Nenad Manevski ${ }^{1}$
}

UCB Celltech UK, Slough, United Kingdom

Received May 8, 2020; accepted August 7, 2020

\begin{abstract}
Although intestinal metabolism plays an important role in drug disposition, early predictions of human outcomes are challenging, in part because of limitations of available in vitro models. To address this, we have evaluated three in vitro models of human intestine (microsomes, permeabilized enterocytes, and cryopreserved intestinal mucosal epithelium) as tools to assess intestinal metabolism and estimate the fraction escaping gut metabolism $\left(f_{\mathrm{g}}\right)$ in drug discovery. The models were tested with a chemically diverse set of 32 compounds, including substrates for oxidoreductive, hydrolytic, and conjugative enzymes. Liquid chromatography-high-resolution mass spectrometry was used to quantify substrate disappearance [intrinsic clearance $\left.\left(\mathrm{CL}_{\text {int }}\right)\right]$ and qualify metabolite formation (quantitativequalitative bioanalysis). Fraction unbound in the incubation $\left(f_{u, \text { inc }}\right)$ was determined by rapid equilibrium dialysis. Measured in vitro results $\left(\mathrm{CL}_{\text {int }}\right.$ and $\left.\boldsymbol{f}_{\mathrm{u} \text {,inc }}\right)$ were supplemented with literature data [passive Caco-2 apical to basolateral permeability, enterocyte blood flow, and intestinal surface area (A)] and combined using a midazolamcalibrated $Q_{\text {gut }}$ model to predict human $f_{\mathrm{g}}$ values. All three models showed reliable CYP and UDP-glucuronosyltransferase activities, but enterocytes and mucosa may offer advantages for low-clearance
\end{abstract}

compounds and alternative pathways (e.g., sulfation, hydrolases, and flavin-containing monooxigenases). Early predictions of human $f_{\mathrm{g}}$ values were acceptable for the high- $f_{\mathrm{g}}$ compounds (arbitrarily $f_{\mathrm{g}}>0.7$ ). However, predictions of low- and moderate- $f_{\mathrm{g}}$ values (arbitrarily $f_{\mathrm{g}}<$ 0.7 ) remain challenging, indicating that further evaluation is needed (e.g., saturation effects and impact of transporters) but not immediate compound avoidance. Results suggest that tested models offer an additional value in drug discovery, especially for drug design and chemotype evaluation.

\section{SIGNIFICANCE STATEMENT}

We found that cellular models of the human gut (permeabilized enterocytes and cryopreserved intestinal mucosa) offer an alternative to and potential advantage over intestinal microsomes in studies of drug metabolism, particularly for low-clearance compounds and alternative pathways (e.g., sulfation, hydrolases, and flavin-containing monooxigenases). The predictivity of human fraction escaping gut metabolism for common CYP and UDP-glucuronosyltransferase substrates based on the $Q_{\text {gut }}$ model is still limited, however, and appropriate further evaluation is recommended.

\section{Introduction}

Metabolism of the intestinal wall plays an important role in the presystemic disposition of oral drugs, including first-pass elimination (Hall et al., 1999; Jones et al., 2016; Peters et al., 2016; Yau et al., 2017), drug interactions (Tachibana et al., 2010; Alqahtani et al., 2018; Yamada et al., 2020), and bioactivation (Dalvie et al., 2008; Yang et al., 2019). Early evaluation of the fraction escaping gut metabolism $\left(f_{\mathrm{g}}\right)$, a significant contributor to oral bioavailability $(F)$ together with fraction absorbed $\left(f_{\mathrm{a}}\right)$ and fraction escaping liver metabolism $\left(f_{\mathrm{h}}\right)$ $\left(F=f_{a} \cdot f_{g} \cdot f_{h}\right)$, is particularly relevant in drug discovery settings, both for predictions of human efficacious dose (Gertz et al., 2010; Peters

This work and its authors were funded by UCB CellTech UK, 216 Bath Rd., Slough SL1 3WE, United Kingdom.

${ }^{1}$ Current affiliation: F. Hoffmann-La Roche Ltd., Basel, Switzerland.

https://doi.org/10.1124/dmd.120.000111. et al., 2016) and evaluation of prodrugs (Williams et al., 2011; Nishimuta et al., 2014; Takahashi et al., 2019). Preferably, human intestinal drug metabolism and corresponding $f_{\mathrm{g}}$ values could be evaluated in vitro, reducing the unnecessary animal testing [3R; EMA/ CHMP/CVMP/JEG-3Rs/450091/2012; (Burden et al., 2015)] and accelerating drug discovery.

Despite the need for an early and accurate evaluation of intestinal drug metabolism, common in vitro models, such as intestinal microsomes and tissue slices, present drug discovery scientists with a difficult choice between a "too simple" and "too complex" model (Fig. 1). Intestinal microsomes generally capture CYP (Gertz et al., 2010) and UDPglucuronosyltransferase (UGT) activities (Cubitt et al., 2009; Kosaka et al., 2011; Gill et al., 2012) but lack many cytosolic [e.g., sulfotransferases, aldehyde oxidase (AO), and various hydrolyses], mitochondrial (e.g., monoamineoxidase), and often even soluble endoplasmic enzymes [carboxylesterases (CESs) (Wang et al., 2018)]. Moreover, a complex process of gut tissue procurement and subcellular

ABBREVIATIONS: $\mathrm{A} \rightarrow \mathrm{B}$, apical to basolateral; $A_{\text {int }}$, intestinal surface area; $\mathrm{AO}$, aldehyde oxidase; $\mathrm{CES}$, carboxylesterase; $\mathrm{Cl}$, confidence interval; $\mathrm{CL}_{\text {int }}$, intrinsic clearance; $f_{\mathrm{a}}$, fraction absorbed; $f_{\mathrm{g}}$, fraction escaping gut metabolism; $f_{\mathrm{u}, \text { inc }}$, fraction unbound in the incubation; FMO, flavincontaining monooxygenase; GMFE, geometric mean fold error; HRMS, high-resolution MS; LC, liquid chromatography; MS, mass spectrometry; $P_{\text {app }}$, apparent permeability; PBPK, physiologically based PK; P-gp, P-glycoprotein; PK, pharmacokinetics; $Q_{\text {ent }}$, enterocytic blood flow; QuanQual, quantitative-qualitative bioanalysis; RED, rapid equilibrium dialysis; UDPGA, uridine 5'-diphosphoglucuronic acid trisodium salt; UGT, UDPglucuronosyltransferase. 


\section{Microsomes}

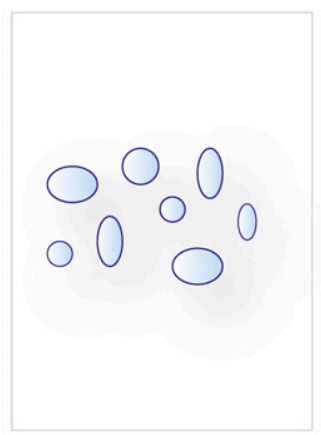

\section{Permeabilized \\ Enterocytes}

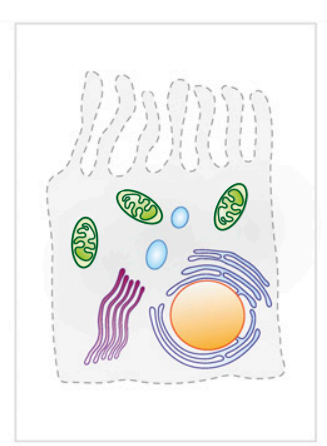

\section{Intestinal \\ Mucosa}

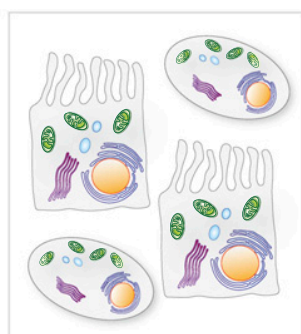

cells prepared from intestinal mucosa
Tissue

slices

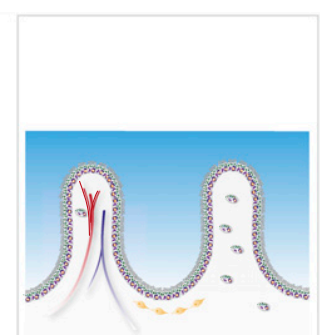

tissue slices with intact intestinal wall

\section{Model complexity}

\section{Drug-metabolizing enzymes}

\section{Donor pooling}

Assay setup
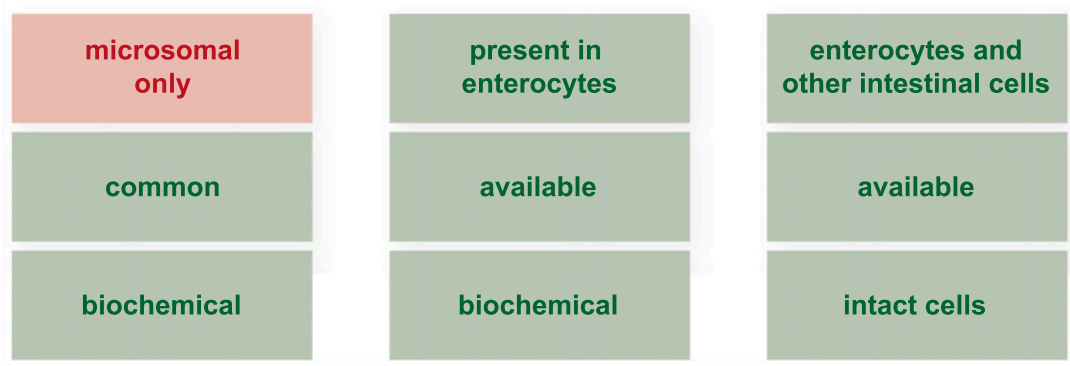

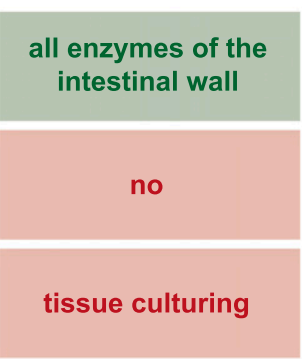

all enzymes of the intestinal wall

Fig. 1. An overview of available in vitro models of the human intestine. fractionation may result in enzyme inactivation (Mohri and Uesawa, 2001; Hatley et al., 2017a,b), leading to underprediction of metabolic clearance and batch-to-batch variability. In contrast to microsomes, intestinal precision-cut tissue slices offer preserved tissue architecture and a complete set of drug-metabolizing enzymes (van de Kerkhof et al., 2006, 2008; de Graaf et al., 2010). Recent advances in organotypic three-dimensional cell cultures-for example, intestinal organoids ( $\mathrm{Lu}$ et al., 2017; Almeqdadi et al., 2019) and gut-on-a-chip systems (Guo et al., 2018) — are equally promising. However, the enduring challenges of ethical procurement of fresh human tissue (Allen et al., 2010), tissue slicing and culturing techniques ( $\mathrm{Li}$ et al., 2016), and interindividual variability (Quignot et al., 2019) render these models better suited for indepth mechanistic studies with a single compound rather than chemotype evaluations performed in drug discovery.

We hypothesized that emerging cellular models of the human gut like primary enterocytes (Ho et al., 2017; Wong et al., 2018) and intestinal mucosal epithelium (Li et al., 2018a) may bridge the gap and offer an in vitro model of "intermediate complexity" suitable for drug discovery applications (Fig. 1) similar to cryopreserved hepatocytes as representative model of human liver (Hengstler et al., 2000; Li, 2007). Although they can potentially capture relevant biology of drug-metabolizing enzymes in the gut wall, these models offer diminished interindividual variability through donor pooling and can be used in automated screening assays (Jacobson et al., 2007). To test this hypothesis, we have assayed 32 test compounds (with versatile biotransformation pathways and a range of human observed $f_{\mathrm{g}}$ values) in human intestinal microsomes, permeabilized and co-factor supplemented MetMax human enterocytes ( $\mathrm{Li}$ et al., 2018b), and cryopreserved human intestinal mucosal epithelium (Li et al., 2018a). Permeabilized human enterocytes (MetMax) were selected based on reported higher activities of several drug-metabolizing enzymes compared with corresponding conventional (nonpermeabilized) enterocytes ( $\mathrm{Li}$ et al., 2018b) and a relatively simple biochemical assay format that resembles microsomal incubations ( $\mathrm{Li}$ et al., 2018b). Cryopreserved intestinal mucosa was included in our evaluation because of a presence of several key intestinal cell types ( $\mathrm{Li}$ et al., 2018a), including enterocytes, goblet cells, enteroendocrine paneth, and microfold cells, potentially broadening the scope of available drug-metabolizing enzymes and approaching level of complexity only available in organotypic gut models (Maresca et al., 2018). As a differentiation compared with intestinal mucosa, permeabilized enterocytes offer an increased cellular permeability and biochemical assay environment (i.e., no need for cell counting and culturing) ( $\mathrm{Li}$ et al., 2018a,c), possibly enabling drug metabolism studies with poorly permeable and cytotoxic compounds (e.g., cancer chemotherapeutics).

To enable simultaneous insight into both quantitative disappearance of the parent drug and qualitative appearance of drug metabolites (QuanQual), all study samples were analyzed with liquid chromatography (LC)-high-resolution mass spectrometry (HRMS) analytical methods. In addition to evaluation of intrinsic clearance $\left(\mathrm{CL}_{\mathrm{int}}\right)$ and biotransformation pathways, a midazolam-calibrated $\mathrm{Q}_{\text {gut }}$ model (Yang et al., 2007; Gertz et al., 2010) was used for early predictions of human $f_{\mathrm{g}}$ values. Similarities and differences between available in vitro models are discussed, enabling an informed approach to intestinal metabolism evaluation in drug discovery settings.

\section{Materials and Methods}

\section{Reagents and Chemicals}

Uridine 5'-diphosphoglucuronic acid trisodium salt (UDPGA), NADPH, alamethicin from Trichoderma viride, adenosine 3-phosphate 5-phosphosulfate, diclofenac sodium salt, midazolam hydrochloride, felodipine, $S$-(+)-mephenytoin, raloxifene hydrochloride, lovastatin, nisoldipine, nifedipine, methadone, quinidine, 
testosterone, benzydamine hydrochloride, cisapride, cyclosporin, terbutaline, sildenafil, verapamil, trazodone, atorvastatin, simvastatin, buspirone, rifabutin, saquinavir, terfenadine, zolpidem, repaglinide, indinavir, alprazolam, carbazeran, enalapril, ramipril, and dabigatran etexilate were purchased from Sigma-Aldrich (St. Louis, MO). NADPH-regenerating system containing NADP ${ }^{+}$, glucose-6phosphate, and glucose-6-phosphate dehydrogenase was purchased from Corning (Wiesbaden, Germany). Organic solvents were of LC-mass spectrometry (MS) or higher quality grade and were acquired from VWR International (Radnor, PA) or Fisher Scientific UK Ltd (Loughborough, UK). Purified water was obtained from Milli-Q Integral 5 Water Purification System (Merck KGaA, Darmstadt, Germany). Phosphate buffer, HQM Hepatocyte/Enterocyte Incubation Medium, and Co-Factor $\mathrm{N}$ for MetMax were purchased from In Vitro ADMET Laboratories (Columbia, MD).

\section{In Vitro Models}

Human intestinal microsomes (lot GYC; mixed-sex, $n=6$; from upper third of the intestine, mostly duodenum) were purchased from BioreclamationIVT (Baltimore, MD) and stored at $-80^{\circ} \mathrm{C}$ until use. Permeabilized human enterocytes (I PHEX MetMax-B, High Performance Pooled Human Enterocytes Phase I/II, lot PHEX3107; mixed-sex, $n=10$; prepared from the duodenum, jejunum, and ileum; stored in $-80^{\circ} \mathrm{C}$ ) and cryopreserved human intestinal mucosal epithelium (PCHIM pooled; lot PCHIM6031; mixed-sex, $n=5$; prepared from the jejunum; stored in liquid nitrogen) were supplied by In Vitro ADMET Laboratories. Certificates of Analysis stated that CYP3A4-mediated rates of testosterone hydroxylation (formation of $6 \beta$-hydroxytestosterone at $200 \mu \mathrm{M}$ of the substrate) were 163,367 , and $612 \mathrm{pmol} / \mathrm{min}$ per milligram or $10^{6}$ cells in intestinal microsomes, permeabilized enterocytes, and cryopreserved mucosa, respectively. Reported rates of 7-hydroxycoumarine glucuronidation were 147, 81, and 62 $\mathrm{pmol} / \mathrm{min}$ per milligram and $10^{6}$ cells in intestinal microsomes, permeabilized enterocytes, and cryopreserved mucosa, respectively. At the time when functional experiments were performed, knowledge of enzyme and transporter protein expression in these models was unknown.

\section{In Vitro Assays}

Incubations with In Vitro Models of the Human Gut Wall. Incubations with human intestinal microsomes were performed with $1 \mathrm{mg} / \mathrm{ml}$ of microsomal protein and NADPH-regenerating system (containing $5.5 \mathrm{mM} \mathrm{MgCl}_{2}$ ) in $100 \mathrm{mM}$ phosphate buffer ( $\mathrm{pH}$ 7.4). For compounds that predominantly undergo glucuronidation, microsomes were preincubated on ice water for 20 minutes with a pore-forming peptide, alamethicin (at a concentration equivalent to 5\% microsomal protein) (Fisher et al., 2000), and incubations were supplemented with UDPGA $(2.5 \mathrm{mM})$. Optimization tests showed that preincubation of microsomes with alamethicin, which is beneficial for optimal UGT activity (Walsky et al., 2012), slightly diminished CYP activities, leading to variability of low-clearance CYP substrates. Therefore, the inclusion of alamethicin and UDPGA was dependent on the compound used and its predominant biotransformation pathway. UDPGA was included for experiments involving testosterone, diclofenac, and raloxifene. Incubations with permeabilized human enterocytes were performed with 500,000 cells $/ \mathrm{ml}$ and Co-Factor $\mathrm{N}$ for MetMax (a mixture of enzyme cofactors) in HQM Hepatocyte/Enterocyte Incubation Medium (Li et al., 2018b). Incubations with intestinal mucosa were performed with $1 \mathrm{mg} / \mathrm{ml}$ total protein (determined by manufacturer) in HQM Hepatocyte/ Enterocyte Incubation Medium ( $\mathrm{Li}$ et al., 2018a). Incubations contained a maximum of $0.5 \%$ acetonitrile and $0.1 \%$ of DMSO.

All incubations were carried out at $37^{\circ} \mathrm{C}$ with continuous shaking at $700 \mathrm{rpm}$ (Eppendorf ThermoMixer C; Thermo Fisher Scientific, Bremen, Germany). Determination of $\mathrm{CL}_{\text {int }}$ and analysis of biotransformation pathways were performed at $1 \mu \mathrm{M}$ final substrate concentration, and incubation time was 1-60 minutes (six time points). Saturation and enzyme kinetic experiments with indinavir and raloxifene were performed at $0.1-100 \mu \mathrm{M}$ (seven to eight concentrations) for 10 minutes. Typically, incubations were performed in a 96-deep well plates with temporal sampling and an incubation volume of $50 \mu \mathrm{l}$ per individual time point. Reactions were prewarmed in an incubator for 5 minutes and initiated with the addition of test substrate. After the required time, reactions were terminated by mixing one volume of incubation mixture with two volumes of ice-cold acetonitrile (containing internal standard) and one volume of ice-cold water. Terminated reactions were placed in a fridge for 20 minutes and then centrifuged for 10 minutes at $4^{\circ} \mathrm{C}$ and $3000 \mathrm{rpm}$. Aliquots of the supernatants were separated from the pellets using a Microlab STAR liquid handling system (Hamilton Robotics Inc., NV) and analyzed by LC-HRMS. All incubations were performed in at least two replications $(n=2-6)$, with midazolam as a positive control.

Determination of Fraction Unbound in the Incubation. The rapid equilibrium dialysis (RED) method (ThermoFisher Scientific, Rockford, IL) was used to determine the degree of nonspecific binding of the compounds to intestinal models during in vitro incubations. Incubations were carried out according to manufacturer's instructions at $37^{\circ} \mathrm{C}$ for 4 hours with $10 \mu \mathrm{M}$ of the test compound (compound concentration was increased compared with functional assays to enable bioanalysis of highly bound compound). Protein or cell concentrations of intestinal models were the same as in the functional assays. Prior to fraction unbound in the incubation $\left(f_{\text {u,inc }}\right)$ determinations, enzymes were inactivated by 48 -hour preincubation at $5^{\circ} \mathrm{C}$, and enzyme inactivity was confirmed by the lack of midazolam metabolite formation after 4 hours of incubation. For highly bound compound, readers are also advised to evaluate longer equilibration times and/or alternative methods (Chen et al., 2019).

\section{Analytical Methods}

Samples were analyzed by LC-HRMS to monitor for both disappearance of test compound and appearance of drug metabolites (QuanQual approach). LC-HRMS system consisted of PAL autosampler (set to $8^{\circ} \mathrm{C}$; CTC Analytics AG, Zwingen, Switzerland), Accela 1250 liquid chromatograph (Thermo Fisher Scientific), MistraSwitch thermostated column compartment (set to $50^{\circ} \mathrm{C}$; MayLab Analytical Instruments $\mathrm{GmbH}$, Germany), and Q Exactive orbitrap mass spectrometer (Thermo Fisher Scientific). Two analytical methods were used: 1) a 5-minute LC gradient with HRMS acquisition for QuanQual analysis using the Phenomex Kinetex C18 column $(50 \times 2.1 \mathrm{~mm}, 5 \mu \mathrm{m})$ (Phenomenex, Inc., Macclesfield, United Kingdom) and 2) a 15-minute LC gradient for metabolite identification with HRMS and data-dependent MS/MS acquisition using a Phenomenex Kinetex C18 column $(100 \times 3.0 \mathrm{~mm}, 2.6 \mu \mathrm{m})$. Both methods used either $0.1 \%$ formic acid in water (low $\mathrm{pH}$ conditions) or $10 \mathrm{mM}$ ammonium bicarbonate, $\mathrm{pH} 8.5$ (high $\mathrm{pH}$ conditions), as eluent $\mathrm{A}$ and $0.1 \%$ formic acid in acetonitrile as eluent $\mathrm{B}$. The $\mathrm{LC}$ gradient for the 5-minute method was $0-0.4$ minutes, $5 \% \mathrm{~B}$; 0.4-2.5 minutes, $5 \% \rightarrow 95 \% \mathrm{~B} ; 2.5-4.5$ minutes, $95 \% \mathrm{~B} ; 4.5-4.6$ minutes, $95 \rightarrow 5 \% \mathrm{~B}$; and $4.6-5.6$ minutes, $5 \% \mathrm{~B}$. The LC gradient for the 15 -minute method was $0-0.8$ minutes, $5 \% \mathrm{~B}$; $0.8-12.0$ minutes, $5 \rightarrow 95 \% \mathrm{~B} ; 12.0-13.0$ minutes, $95 \% \mathrm{~B}$; $13.0-13.1$ minutes, $95 \rightarrow 5 \% \mathrm{~B}$; and $13.1-15.0$ minutes, $5 \% \mathrm{~B}$. The flow rate of the mobile phase was $0.6 \mathrm{ml} / \mathrm{min}$ for the 5 -minute method and $0.5 \mathrm{ml} / \mathrm{min}$ for the 15 -minute method with an injection volume of $10 \mu \mathrm{l}$.

\section{Data Analysis}

Chemical structures and biotransformation schemes were prepared with ACD/ ChemSketch 2018.1 (ACD Laboratories). Graphs, linear and nonlinear regression, and statistical analysis were performed with GraphPad Prism 8.1 (GraphPad Software)

Determination of Intestinal $\mathbf{C L}_{\text {int }} \cdot \mathbf{C L}_{\text {int }}$ (microliters per minute per milligram of total protein or microliters per minute per $10^{6}$ cells) was determined by following the depletion of test compound over incubation time. Compound elimination rate constant $\left(k_{\mathrm{el}} ; 1 / \mathrm{min}\right)$ was determined by using nonlinear regression and monoexponential decay equation (GraphPad Prism 8.1; GraphPad Software, San Diego CA). $\mathrm{CL}_{\text {int }}$ was calculated as (eq. 1):

$$
C L_{\text {int }}=k_{\text {el }} \frac{V_{\text {inc }}}{m(\text { enzyme source })}
$$

In which m(enzyme source) represents either total amount of protein (microsomal and mucosal incubations) or total number of cells (enterocyte incubations) used in the assay, and $V_{\text {inc }}$ represents the volume of incubation (microliters). Estimated lower limit of quantification for $\mathrm{CL}_{\text {int }}$ determination was $1 \mu \mathrm{l} / \mathrm{min}$ per $10^{6}$ cells or $1 \mu \mathrm{l} / \mathrm{min}$ per milligram protein, corresponding to $97 \%$ of test compound remaining after a 60 -minute incubation. Results are presented as mean \pm S.E.

Analysis of Intestinal Biotransformation Pathways. For drug metabolite profiling, acquired LC-HRMS raw data were processed by Compound Discoverer 3.1 (Thermo Fisher Scientific) using a targeted workflow. Theoretical drug metabolites were predicted from various oxidoreductive (oxidation, desaturation, 
hydration, hydrolysis, dealkylation, etc.) and conjugative (glucuronidation, sulfation, glutathione conjugation, etc.) biotransformation pathways, including all combinations up to three steps, and generated mass ions were searched against experimentally obtained data. Metabolite identity was established by a combination of mass tolerance $(<10 \mathrm{ppm})$, absence in blank incubations, kinetics of metabolite formation, matching isotope patterns, and, when appropriate, analysis of MS/MS data. Because the parent compound and its metabolites may show different ionization responses in mass spectrometer, obtained metabolite data are qualitative or semiquantitative at best (Blanz et al., 2017).

Prediction of Human fg Values Based on In Vitro Data. Human $f_{\mathrm{g}}$ values were predicted based on midazolam-calibrated $Q_{\text {gut }}$ model (Rostami-Hodjegan and Tucker, 2002; Yang et al., 2007; Gertz et al., 2010). In addition to $\mathrm{CL}_{\text {int }}$ values experimentally measured in this work, $Q_{\text {gut }}$ model requires measurements of intestinal permeability [either apparent permeability $\left(P_{\text {app}}\right.$; nanometers per second) or effective permeability ( $P_{\text {eff; }}$; micrometers per second)], intestinal surface area $\left(A_{\text {int }}\right.$; squared meter), and enterocytic blood flow $\left(Q_{\text {ent }}\right.$; liters per hour). Because measured in vivo $P_{\text {eff }}$ values are rarely available in drug discovery settings, and conversion between $P_{\text {app }}$ and $P_{\text {eff }}$ could introduce additional errors (Gertz et al., 2010), we have directly used literature in vitro $P_{\text {app }}$ values (nanometers per second) from Caco-2 $\mathrm{A} \rightarrow \mathrm{B}$ passive permeability assays (Wu et al., 2000; Polli et al., 2001; Hochman et al., 2004; Gertz et al., 2010; Lee et al., 2017). Literature Caco-2 $P_{\text {app }}$ values were supplemented with experimentally measured values for ramipril, enalapril, terbutaline, terfenadine, raloxifene, benzydamine, and carbazeran (Cyprotex, Alderley Park, UK). Because dabigatran etexilate is known to undergo esterase-mediated hydrolysis in the Caco-2 cells (Ishiguro et al., 2014), we have used the passive $P_{\text {app }}$ value obtained in the optimized assay, which was performed in the presence of both CES inhibitor [bis(p-nitrophenyl) phosphate)] and P-gp inhibitor (cyclosporin A) (Ishiguro et al., 2014). Average values of $200 \mathrm{~m}^{2}$ for $A_{\text {int }}$ (Gertz et al., 2010) and $181 / \mathrm{h}$ for $Q_{\text {ent }}$ were used (Granger et al., 1980).

Permeability clearance $\left(\mathrm{CL}_{\text {perm }}\right)$, a hybrid parameter of $P_{\text {app }}$ and $A_{\text {int }}$, was calculated as (eq. 2):

$$
C L_{\text {perm }}=P_{\text {app }} \cdot A_{\text {int }}
$$

$\mathrm{CL}_{\text {perm }}$ and $Q_{\text {ent }}$ were combined to form a $Q_{\text {gut }}$, a hybrid parameter of permeability through the enterocyte membrane and $Q_{\text {ent }}$ (eq. 3):

$$
Q_{\text {gut }}=\frac{C L_{\text {perm }} \cdot Q_{\text {ent }}}{Q_{\text {ent }}+C L_{\text {perm }}}
$$

The $f_{\mathrm{g}}$ is then calculated by the following equation (eq. 4):

$$
f_{g}=\frac{Q_{\text {gut }}}{Q_{\text {gut }}+f_{u, g u t} \frac{C L_{\text {int }}}{f_{u, i n c}}}
$$

In which $f_{\mathrm{u}, \text { gut }}$ and $f_{\mathrm{u} \text {,inc }}$ represent the fraction unbound in the gut and enterocyte incubations, respectively. The errors of $\mathrm{CL}_{\text {int }}$ measurements were propagated into calculated $f_{\mathrm{g}}$ values by using the following equation (eq. 5) (Taylor, 1996):

$$
\delta f_{g}=\frac{d f_{g}}{d C L_{i n t}} \delta C L_{\text {int }}
$$

In which $\delta f_{\mathrm{g}}$ and $\delta \mathrm{CL}_{\mathrm{int}}$ are errors of $f_{\mathrm{g}}$ and $\mathrm{CL}_{\text {int }}$, respectively, and $\mathrm{d} f_{\mathrm{g}} / \mathrm{dCL}_{\mathrm{int}}$ is the first derivative of the $f_{\mathrm{g}}$ function over $\mathrm{CL}_{\text {int }}$.

To enable direct comparison of $f_{\mathrm{g}}$ predictivity between three intestinal models (intestinal microsomes, permeabilized enterocytes, and mucosa) and account for batch-to-batch variations, calculated $f_{\mathrm{g}}$ values were calibrated to in vivo observed $f_{\mathrm{g}}$ value of midazolam [0.55 \pm 0.04; mean \pm S.E.; $n=6$; (Paine, 1996; Galetin et al., 2006; Gertz et al., 2008, 2010; Nishimuta et al., 2011; Karlsson et al., 2013)]. Midazolam calibration resulted in microsomal-, enterocytic-, and mucosal-predicted $f_{\mathrm{g}}$ values to be corrected $+9.1 \%,+18.2 \%$, and $-12.7 \%$, respectively. Minimal and maximal predicted $f_{\mathrm{g}}$ values were arbitrarily set to 0.01 and 1 , respectively.

Evaluation of Observed Human $\boldsymbol{f}_{\mathrm{g}}$ Values. Observed in vivo human $f_{\mathrm{g}}$ values were obtained from the literature for alprazolam, atorvastatin, buspirone, cisapride, cyclosporine, felodipine, indinavir, lovastatin, methadone, midazolam, nifedipine, nisoldipine, quinidine, raloxifene, repaglinide, rifabutin, saquinavir, sildenafil, simvastatin, tacrolimus, terfenadine, trazodone, triazolam, verapamil, and zolpidem (Paine et al., 1996; Galetin et al., 2006; Yang et al., 2007; Gertz et al., 2008, 2010; Mizuma, 2009; Nishimuta et al., 2011; Karlsson et al., 2013) (Table 1). The majority of human $f_{\mathrm{g}}$ values was estimated through interpretation of clinical PK results (e.g., intravenous and oral drug administration) and corresponding in vitro data. A smaller subset of observed human $f_{\mathrm{g}}$ values, including CYP3A4 substrates midazolam, felodipine, buspirone, quinidine, and lovastatin, were obtained via the "grapefruit juice method" (Gertz et al., 2008; Hanley et al., 2011). When multiple estimates of observed $f_{\mathrm{g}}$ values were published, the arithmetic mean \pm S.D. was used for comparisons with predicted $f_{\mathrm{g}}$ values. Because clinical PK results may be influenced by a number of variables, including subject number, age, sex, ethnicity, drug dose, disease state, and polymorphisms of enzymes and transporters, readers should note that reported human $f_{\mathrm{g}}$ values are estimates at the current level of knowledge. Although more sophisticated meta-analyses of clinical data, including interstudy and interindividual variability and weighted means, were performed previously (Gertz et al., 2008), this was beyond the scope of current work. If literature values were not available, observed $f_{\mathrm{g}}$ values were estimated from available clinical PK data and complementary in vitro data using the following equation (eq. 6):

$$
f_{g}=\frac{F}{f_{a} \cdot\left(1-\frac{Q_{h}}{C L_{b l o o d}}\right)}
$$

In which $F$ is the absolute bioavailability of the drug (fraction $0-1$ ), $f_{\mathrm{a}}$ is the fraction of the dose absorbed (fraction $0-1$ ), $Q_{\mathrm{h}}$ is the human liver blood flow [1450 ml/min for a 70-kg individual (Davies and Morris, 1993)], and $C L_{\mathrm{blood}}$ is the systemic clearance of the drug (assumption $C L_{\text {blood }} \approx C L_{\text {liver }}$ ). As an example, clinical PK parameters for diclofenac were taken from (Willis et al., 1979; Hinz et al., 2005), and $f_{\mathrm{a}}$ was assumed to be 1 because of high Caco- $2 P_{\text {app }}$ value ( $>100$ $\mathrm{nm} / \mathrm{s}$ ). Similar evaluations were performed for enalapril (Davies et al., 1984), raloxifene (Mizuma, 2009), testosterone (Tauber et al., 1986), dabigatran etexilate (Blech et al., 2008; Ishiguro et al., 2014), and benzydamine (Baldock et al., 1991). Human $f_{\mathrm{g}}$ values of carbazeran $[\mathrm{F}<5 \%$ (Kaye et al., 1984)], $S$-mephenytoin $[\mathrm{F}<$ $5 \%$ and large interindividual variability (Wedlund et al., 1985)], and terbutaline could not be estimated based on available data.

Comparison of Observed and Predicted Human $f_{\mathrm{g}}$ Values. Observed and predicted human $f_{\mathrm{g}}$ values were compared based on geometric mean fold error (GMFE). Furthermore, based on the range of observed human $f_{\mathrm{g}}$ values, compounds were arbitrarily classified into low $\left(f_{\mathrm{g}} 0-0.3\right)$, moderate $\left(f_{\mathrm{g}}\right.$ $0.31-0.70)$, and high $\left(f_{\mathrm{g}} 0.71-1.0\right)$ groups. GMFE values, classification success (based on predicted $f_{\mathrm{g}}$ values), and prediction bias were calculated for each group.

Saturation and Enzyme Kinetic Experiments. To assess the effects of enzyme saturation, the apparent $\mathrm{CL}_{\text {int }}$ for indinavir and raloxifene was measured across the range of substrate concentrations $(0.1-100 \mu \mathrm{M})$ in human permeabilized enterocytes. The sum of LC-HRMS peak areas for all observed metabolites was used to estimate the apparent $K_{\mathrm{m}}$ values. The velocity of the enzymatic reaction $(v)$ was plotted against the substrate concentration $([S])$, and data were fitted to either Michaelis-Menten $\left(v=\frac{V_{\max }[S]}{K_{m}+[S]}\right)$ or substrate inhibition equations $\left(v=\frac{V_{\text {max }}[S]}{K_{m}+[S] 1+\frac{[S]}{K_{\mathrm{si}}}}\right)$, in which $K_{\mathrm{m}}$ is the Michaelis-Menten constant, $V_{\max }$ is the limiting reaction velocity, and $K_{\mathrm{si}}$ is the substrate inhibition constant. Because drug metabolites were not quantified with authentic standards but rather observed with QuanQual bioanalytical approach, $V_{\max }$ values are qualitative and expressed at the metabolite peak area per minute $/ 10^{6}$ cells. The $K_{\mathrm{m}}$ values were also estimated based on the substrate disappearance data. Observed elimination rate constants $\left(k_{\mathrm{el}}, 1 / \mathrm{min}\right)$ were plotted against the substrate concentration ([S], micromolars), and data were fitted to the following equation: $k_{e l}=k_{e l, \text { max }}\left(1-\frac{[S]}{K_{m}+[S]}\right)$, in which $k_{\mathrm{el}, \max }$ is the theoretical maximal $k_{\mathrm{el}}$ at an infinitesimally low concentration of substrate (Obach and Reed-Hagen, 2002).

\section{Results}

Selection of the Test Compounds. Our 32 test compounds were selected based on a range of observed human $f_{\mathrm{g}}$ values as well as different biotransformation pathways and diverse drug-like properties (Table 1). Most of the previously reported observed human $f_{\mathrm{g}}$ values were estimated through integration of clinical PK results (e.g., intravenous and oral drug 
TABLE 1

Test compounds used in this study $(n=32)$, sorted by the increasing avg. observed human $f_{\mathrm{g}}$ value

Compounds were selected based on a wide range of observed $\mathrm{f}_{\mathrm{g}}$ values, different biotransformation pathways, and physicochemical properties. Maximum dose, aqueous solubility, and logD values were reported from (Benet et al., 2011) and other publicly available sources (Drug Bank, https://www.drugbank.ca/; ChEMBL, https://www.ebi.ac.uk/chembl/).

\begin{tabular}{|c|c|c|c|c|c|c|c|c|}
\hline Test Compound & $\begin{array}{l}\text { Main Biotransformation } \\
\text { Pathways }\end{array}$ & $\begin{array}{l}\text { Drug-Metabolizing } \\
\text { Enzymes }\end{array}$ & $\begin{array}{l}\text { Maximum } \\
\text { Dose }(\mathrm{mg})\end{array}$ & $\begin{array}{l}\text { Aqueous } \\
\text { Solubility (mg/ } \\
\text { ml) }\end{array}$ & $\begin{array}{c}\operatorname{LogD} \\
(\mathrm{pH} 7.4)\end{array}$ & $\begin{array}{l}\text { Caco-2 } \\
P_{\text {app }} \mathrm{A} \rightarrow \mathrm{B} \\
\text { Passive } \\
(\mathrm{nm} / \mathrm{s})\end{array}$ & $\begin{array}{l}\text { Human Observed } \\
f_{\mathrm{g}} \text { Value (Mean } \pm \\
\text { S.D.) }\end{array}$ & References \\
\hline Raloxifene & $\begin{array}{l}\text { Glucuronidation and } \\
\text { sulfation }\end{array}$ & UGT1A family & 60 & 0.013 & 3.40 & $221^{a}$ & 0.05 & Mizuma, 2009 \\
\hline Testosterone & $\begin{array}{c}\text { Glucuronidation, } \\
\text { oxidation }\end{array}$ & CYP3A4/UGT & 40 & 0.023 & 3.18 & 723 & $0.05^{b}$ & $\begin{array}{c}\text { Tauber et al., 1986; Buur and Mork, } \\
1992\end{array}$ \\
\hline $\begin{array}{r}\text { Dabigatran } \\
\text { etexilate }\end{array}$ & Hydrolysis & CES1/CES2 & 110 & 1.8 & 3.08 & 290 & $0.05^{b}$ & $\begin{array}{l}\text { Blech et al., 2008; Ishiguro et al., } \\
\text { 2014; Laizure et al., } 2014\end{array}$ \\
\hline Lovastatin & Oxidation & $\begin{array}{c}\text { CYP3A4/ } \\
\text { CYP2C/ } \\
\text { CYP2D6/UGT }\end{array}$ & 40 & 0.0004 & 4.30 & 145 & $0.07^{c}$ & Yang et al., 2007; Gertz et al., 2008 \\
\hline Nisoldipine & Oxidation & CYP3A4 & 34 & 0.0057 & 4.96 & $>200$ & 0.11 & Gertz et al., 2010 \\
\hline Buspirone & Oxidation & CYP3A4 & 10 & 0.0214 & 1.24 & 254 & $\begin{array}{l}0.18 \pm 0.06 \\
\quad(n=3)^{c}\end{array}$ & $\begin{array}{c}\text { Galetin et al., 2006; Gertz et al., 2008, } \\
2010\end{array}$ \\
\hline Rifabutin & Oxidation & CYP3A4 & 150 & 0.017 & 1.92 & 95 & 0.21 & Gertz et al., 2010 \\
\hline Saquinavir & Oxidation & CYP3A4 & 500 & 0.00247 & 5.05 & 4.00 & $\begin{aligned} 0.28 & \pm 0.16 \\
(n & =3)\end{aligned}$ & $\begin{array}{l}\text { Gertz et al., 2010; Nishimuta et al., } \\
\text { 2011; Karlsson et al., } 2013\end{array}$ \\
\hline Simvastatin & $\begin{array}{l}\text { Oxidation, } \\
\text { glucuronidation }\end{array}$ & $\begin{array}{c}\text { CYP3A4/ } \\
\text { CYP2C/ } \\
\text { CYP2D6/UGT }\end{array}$ & 80 & 0.012 & 4.72 & 68 & $\begin{array}{l}0.29 \pm 0.32 \\
\quad(n=3)\end{array}$ & $\begin{array}{c}\text { Galetin et al., 2006; Gertz et al., 2010; } \\
\text { Nishimuta et al., } 2011\end{array}$ \\
\hline Atorvastatin & $\begin{array}{l}\text { Oxidation, } \\
\text { glucuronidation }\end{array}$ & $\begin{array}{c}\text { CYP3A4/ } \\
\text { CYP2C/ } \\
\text { CYP2D6/UGT }\end{array}$ & 80 & 0.00063 & 0.74 & 60 & $\begin{array}{l}0.40 \pm 0.16 \\
\quad(n=2)\end{array}$ & Gertz et al., 2008, 2010 \\
\hline Terfenadine & Oxidation & CYP3A4 & 60 & 0.0000963 & 3.60 & $1.73^{a}$ & 0.40 & Gertz et al., 2010 \\
\hline Felodipine & Oxidation & CYP3A4 & 10 & 0.00715 & 4.76 & 42 & $\begin{array}{l}0.42 \pm 0.10 \\
\quad(n=5)^{c}\end{array}$ & $\begin{array}{l}\text { Galetin et al., 2006; Gertz et al., 2008, } \\
\text { 2010; Nishimuta et al., 2011; Karlsson } \\
\text { et al., } 2013\end{array}$ \\
\hline Cyclosporin & Oxidation & CYP3A4 & 100 & $6.6 \times 10^{-9}$ & 2.92 & 5.0 & $\begin{aligned} 0.48 & \pm 0.11 \\
(n & =3)\end{aligned}$ & $\begin{array}{c}\text { Galetin et al., 2006; Gertz et al., 2010; } \\
\text { Karlsson et al., } 2013\end{array}$ \\
\hline Midazolam & Oxidation & CYP3A4 & 3 & 0.00987 & 3.78 & 324 & $\begin{array}{l}0.55 \pm 0.10 \\
\quad(n=6)^{c}\end{array}$ & $\begin{array}{l}\text { Paine et al., 1996; Galetin et al., 2006; } \\
\text { Gertz et al., 2008, 2010; Nishimuta } \\
\text { et al., 2011; Karlsson et al., } 2013\end{array}$ \\
\hline Cisapride & Oxidation & CYP3A4 & 20 & 0.012 & 2.46 & 299 & $\begin{array}{l}0.57 \pm 0.02 \\
\quad(n=2)\end{array}$ & Bohets et al., 2000 \\
\hline Ramipril & Hydrolysis & CES & 10 & 0.039 & 0.81 & $1.37^{a}$ & 0.59 & $\begin{array}{c}\text { van Griensven et al., 1995; Levitt and } \\
\text { Schoemaker, } 2006\end{array}$ \\
\hline Verapamil & Oxidation & $\begin{array}{l}\text { CYP3A4/ } \\
\text { CYP1A2 }\end{array}$ & 120 & 0.00394 & 2.46 & 138 & $\begin{aligned} 0.60 & \pm 0.18 \\
(n & =3)\end{aligned}$ & $\begin{array}{l}\text { Gertz et al., 2010; Nishimuta et al., } \\
\text { 2011; Karlsson et al., } 2013\end{array}$ \\
\hline Sildenafil & Oxidation & CYP3A4 & 100 & 0.433 & 2.45 & 256 & $\begin{array}{l}0.62 \pm 0.11 \\
\quad(n=2)\end{array}$ & Gertz et al., 2010; Karlsson et al., 2013 \\
\hline Nifedipine & Oxidation & $\begin{array}{l}\text { CYP3A4/ } \\
\text { CYP2C9 }\end{array}$ & 20 & 0.0177 & 3.58 & 235 & $\begin{array}{l}0.71 \pm 0.17 \\
\quad(n=4)\end{array}$ & $\begin{array}{l}\text { Galetin et al., 2006; Gertz et al., 2010; } \\
\text { Nishimuta et al., 2011; Karlsson et al., } \\
2013\end{array}$ \\
\hline Methadone & Oxidation & CYP3A4 & 10 & 0.0059 & 2.29 & 219 & 0.78 & Gertz et al., 2010 \\
\hline Diclofenac & Oxidation & CYP2C9/UGT & 50 & 0.0045 & 1.44 & 133 & 0.78 & \\
\hline Zolpidem & Oxidation & CYP3A4 & 10 & 23 & 3.03 & 319 & 0.79 & Gertz et al., 2010 \\
\hline Trazodone & Oxidation & CYP2D6 & 300 & 0.0276 & 2.58 & 242 & 0.83 & Gertz et al., 2010 \\
\hline Repaglinide & Oxidation & $\begin{array}{l}\text { CYP3A4/ } \\
\text { CYP2C8 }\end{array}$ & 2 & 0.0029 & 1.99 & 241 & 0.89 & Gertz et al., 2010 \\
\hline Quinidine & Oxidation & $\begin{array}{l}\text { CYP3A4/ } \\
\text { CYP2D6 }\end{array}$ & 404 & 0.33 & 0.98 & 85 & $\begin{array}{l}0.91 \pm 0.01 \\
\quad(n=4)^{c}\end{array}$ & $\begin{array}{c}\text { Galetin et al., 2006; Gertz et al., 2008, } \\
\text { 2010; Nishimuta et al., } 2011\end{array}$ \\
\hline Indinavir & Oxidation & CYP3A4 & 400 & 0.0482 & 3.43 & 12 & 0.93 & Gertz et al., 2010 \\
\hline Alprazolam & Oxidation & CYP3A4 & 2 & 0.032 & 1.92 & 255 & $\begin{aligned} 0.93 & \pm 0.05 \\
(n & =4)\end{aligned}$ & $\begin{array}{l}\text { Galetin et al., 2006; Yang et al., 2007; } \\
\text { Gertz et al., 2010; Karlsson et al., } 2013\end{array}$ \\
\hline Enalapril & $\begin{array}{l}\text { Hydrolysis, } \\
\text { oxidation }\end{array}$ & Esterase/CYP & 20 & 0.213 & -0.32 & $3.36^{a}$ & 0.97 & Davies et al., 1984 \\
\hline Benzydamine & Oxidation & FMO/CYP & 2 & 0.0491 & 1.65 & $192^{a}$ & 0.98 & Baldock et al., 1991 \\
\hline Carbazeran & Oxidation & $\mathrm{AO} / \mathrm{CYP}$ & NA & 0.0266 & -0.35 & $190^{a}$ & NA & Kaye et al., 1984 \\
\hline$S$-Mephenytoin & Oxidation & CYP2C19 & NA & 0.97 & 1.36 & 346 & NA & Wedlund et al., 1985 \\
\hline Terbutaline & Sulfation & SULT & 5 & 213 & -1.44 & $1.96^{a}$ & NA & \\
\hline
\end{tabular}

NA, data not available; SULT, sulfotransferase.

${ }^{a}$ Caco-2 $P_{\text {app }}$ values (passive; nanometers per second) were experimentally determined at Cyprotex.

${ }^{b} \mathrm{An}$ in-house estimate based on available human data (PK and in vitro)

${ }^{c}$ Some of the observed human $f_{\mathrm{g}}$ values were obtained via the grapefruit juice method.

administration) and corresponding in vitro data (e.g., solubility and permeability). A smaller subset of observed human $f_{\mathrm{g}}$ values for midazolam, felodipine, buspirone, quinidine, and lovastatin were obtained via the "grapefruit juice method," an approach that utilizes
CYP3A4 inhibition of grapefruit juice ingredients (Hanley et al., 2011). Although the majority of human $f_{\mathrm{g}}$ values were obtained from the literature (see Table 1 for references), values for enalapril, raloxifene, testosterone, dabigatran etexilate, and benzydamine were estimated based 
TABLE 2

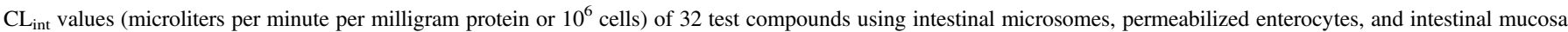
and their respective observed in vivo $f_{\mathrm{g}}$ value

$\mathrm{CL}_{\text {int }}$ values were measured in at least three independent experiments, and results are presented as the mean \pm S.E.

\begin{tabular}{|c|c|c|c|c|}
\hline \multirow[b]{2}{*}{ Compound } & \multirow{2}{*}{$\begin{array}{c}\text { Observed Human } \\
f_{\mathrm{g}} \text { Value } \\
\text { Range } \\
0-1\end{array}$} & Intestinal Microsomes & Permeabilized Enterocytes & \multirow{2}{*}{$\frac{\text { Intestinal Mucosa }}{\mu \mathrm{l} / \mathrm{Min} \text { per } 10^{6} \mathrm{Cells}}$} \\
\hline & & \multicolumn{2}{|c|}{$\mu \mathrm{l} /$ Min per Milligram Protein } & \\
\hline Dabigatran etexilate & 0.05 & $69.5 \pm 3.7$ & $669.4 \pm 15.4$ & $585.2 \pm 8.5$ \\
\hline Raloxifene & 0.05 & $338.0 \pm 22.7$ & $114.2 \pm 19.6$ & $203.0 \pm 20.6$ \\
\hline Testosterone & 0.05 & $95.3 \pm 7.7$ & $545.7 \pm 130.2$ & $612.7 \pm 37.9$ \\
\hline Lovastatin & 0.07 & $83.8 \pm 23.2$ & $186.5 \pm 10.8$ & $243.4 \pm 14.7$ \\
\hline Nisoldipine & 0.11 & $78.1 \pm 7.9$ & $131.8 \pm 19.5$ & $199.3 \pm 13.6$ \\
\hline Buspirone & 0.18 & $5.1 \pm 2.5$ & $7.8 \pm 1.9$ & $19.8 \pm 2.8$ \\
\hline Rifabutin & 0.21 & $2.9 \pm 1.0$ & $16.2 \pm 4.0$ & $2.7 \pm 1.5$ \\
\hline Saquinavir & 0.28 & $140.4 \pm 7.0$ & $357.0 \pm 46.2$ & $60.6 \pm 3.3$ \\
\hline Simvastatin & 0.29 & $\mathrm{ND}^{a}$ & $6.8 \pm 3.3$ & $7.9 \pm 11.1$ \\
\hline Atorvastatin & 0.40 & $1.9 \pm 1.4$ & $3.0 \pm 1.4$ & $\mathrm{ND}^{a}$ \\
\hline Terfenadine & 0.40 & $9.0 \pm 3.1$ & $55.5 \pm 10.1$ & $57.1 \pm 1$ \\
\hline Felodipine & 0.42 & $14.5 \pm 2.1$ & $64.8 \pm 10.6$ & $85.7 \pm 2.6$ \\
\hline Cyclosporin & 0.48 & $10.4 \pm 2.6$ & $18.3 \pm 8.6$ & $56.5 \pm 0.0$ \\
\hline Midazolam & 0.55 & $10.8 \pm 1.1$ & $9.2 \pm 1.3$ & $16.9 \pm 3$ \\
\hline Cisapride & 0.57 & $4.6 \pm 1.5$ & $19.2 \pm 3.3$ & $27.0 \pm 4.1$ \\
\hline Ramipril & 0.59 & $1.4 \pm 0.0$ & $\mathrm{ND}^{a}$ & $\mathrm{ND}^{a}$ \\
\hline Verapamil & 0.60 & $2.1 \pm 1.0$ & $33.6 \pm 4.5$ & $73.6 \pm 11.1$ \\
\hline Sildenafil & 0.62 & $4.7 \pm 1.6$ & $2.2 \pm 2.2$ & $5.0 \pm 7.2$ \\
\hline Nifedipine & 0.71 & $2.9 \pm 2.2$ & $11.1 \pm 2.8$ & $5.9 \pm 1.2$ \\
\hline Diclofenac & 0.78 & $27.7 \pm 3.2$ & $9.9 \pm 3.2$ & $10.4 \pm 0.5$ \\
\hline Methadone & 0.78 & $\mathrm{ND}^{a}$ & $14.8 \pm 1.7$ & $6.3 \pm 0.8$ \\
\hline Zolpidem & 0.79 & $7.0 \pm 2.2$ & $3.1 \pm 2.1$ & $\mathrm{ND}^{a}$ \\
\hline Trazodone & 0.83 & $3.0 \pm 0.0$ & $5.8 \pm 2.4$ & $\mathrm{ND} a$ \\
\hline Repaglinide & 0.89 & $2.2 \pm 1.8$ & $1.2 \pm 3.5$ & $1.3 \pm 0.6$ \\
\hline Quinidine & 0.91 & $\mathrm{ND}^{a}$ & $\mathrm{ND}^{a}$ & $\mathrm{ND}^{a}$ \\
\hline Indinavir & 0.93 & $3.7 \pm 1.4$ & $25.2 \pm 3.7$ & $1.9 \pm 1.0$ \\
\hline Alprazolam & 0.94 & $2.2 \pm 1.2$ & $1.5 \pm 0.7$ & $1.4 \pm 0.6$ \\
\hline Enalapril & 0.97 & $\mathrm{ND}^{a}$ & $\mathrm{ND}^{a}$ & $\mathrm{ND}^{a}$ \\
\hline Benzydamine & 0.98 & $\mathrm{ND}^{a}$ & $28.7 \pm 0.0$ & $18.6 \pm 0.0$ \\
\hline Carbazeran & $\mathrm{NA}^{b}$ & $2.1 \pm 0.8$ & $\mathrm{ND}^{a}$ & $\mathrm{ND}^{a}$ \\
\hline$S$-Mephenytoin & $\mathrm{NA}^{b}$ & $3.4 \pm 0.0$ & $\mathrm{ND}^{a}$ & $\mathrm{ND}^{a}$ \\
\hline Terbutaline & $\mathrm{NA}^{b}$ & $\mathrm{ND}^{a}$ & $\mathrm{ND}^{a}$ & $\mathrm{ND}^{a}$ \\
\hline
\end{tabular}

NA, data not available; ND, not determined.

${ }^{a} \mathrm{CL}_{\text {int }}$ values detected were below the limit of quantification; $1 \mu \mathrm{l} / \mathrm{min}$ per $10^{6}$ cells or $1 \mu \mathrm{l} / \mathrm{min}$ per milligram protein, corresponding to $97 \%$ of substrate remaining after 60 min of incubation. ${ }^{b}$ Insufficient information in the literature to estimate human $f_{\mathrm{g}}$.

on available human PK results and corresponding in vitro data (see Data Analysis for details). The observed $f_{\mathrm{g}}$ values for test compounds ranged from 0.05 to 0.98 , with median and average values of 0.57 and 0.53 , respectively. Whereas most of the test compounds undergo oxidative metabolism (especially by CYP3A4), raloxifene, testosterone, and terbutaline undergo conjugations (glucuronidation and sulfation), and dabigatran etexilate, ramipril, and enalapril are hydrolyzed by hydrolytic enzymes. In addition to CYP-mediated oxidations, benzydamine is predominantly oxidized by FMOs and carbazeran by AO. Regarding the drug-like and physicochemical properties, tested compounds showed a diverse range of values (Table 1) (range; mean \pm S.D.): Caco-2 permeability (1.37-722; $167 \pm 150 \mathrm{~nm} / \mathrm{s}), \operatorname{logD}$ at $\mathrm{pH} 7.4(-1.44-5.05 ; 2.50 \pm 1.65)$, aqueous solubility $(<0.01-213 \mathrm{mg} / \mathrm{ml})$, and maximum dose $(2-500 \mathrm{mg})$.

In Vitro $\mathbf{C L}_{\text {int }}$. The $\mathrm{CL}_{\text {int }}$ values were measured for all 32 test compounds in intestinal microsomes, permeabilized enterocytes, and intestinal mucosa. Experiments were performed at $1 \mu \mathrm{M}$ of substrate and 1-60 minutes of incubation, resembling assay conditions typical for drug discovery. Numerical results are presented in Table 2 and selected graphical examples in Fig. 2 (for all graphs, see Supplemental Figs. 1-6). For test substrates predominantly metabolized by CYPs, the observed rate of metabolism was generally comparable between models, although average values of $\mathrm{CL}_{\text {int }}$ were 2 -fold higher in permeabilized enterocytes and intestinal mucosa (44.74 and $46.46 \mu \mathrm{l} / \mathrm{min}$ per milligram or $10^{6}$ cells, respectively, compared with $20.85 \mu \mathrm{l} / \mathrm{min}$ per milligram in microsomes). Higher observed CYP metabolism in permeabilized enterocytes and intestinal mucosa is aligned with formation rates of $6 \beta$-hydroxytestosterone reported by vendors in the corresponding Certificates of Analysis. However, a few exceptions to this trend were observed, with $S$-mephenytoin and zolpidem showing higher $\mathrm{CL}_{\text {int }}$ in intestinal microsomes. Benzydamine, an FMO substrate (Lang and Rettie, 2000), showed a significantly higher $\mathrm{CL}_{\text {int }}$ in permeabilized enterocytes and mucosa compared with microsomes (Table 2). Carbazeran, a high-turnover substrate for human AO (Kaye et al., 1984), showed very low clearance in all three intestinal models. Test substrates for hydrolytic enzymes, namely dabigatran etexilate, ramipril, and enalapril, showed variable results (Table 2). Although $\mathrm{CL}_{\mathrm{int}}$ of dabigatran etexilate was 10 -fold higher in permeabilized enterocytes and intestinal mucosa, metabolism of ramipril and enalapril was minimal in all three models. Raloxifene and testosterone, test substrates for glucuronidation and sulfation, generally exhibited high $\mathrm{CL}_{\text {int }}$ in all three models (typically $>100 \mu \mathrm{l} / \mathrm{min}$ per milligram or $10^{6}$ cells), although $\mathrm{CL}_{\mathrm{int}}$ of testosterone was still 5-fold higher in permeabilized enterocytes and intestinal mucosa. Although 60-minute incubation time enabled a wide dynamic range of the $\mathrm{CL}_{\text {int }}$ assays, one may consider shorter or longer incubation times depending on the properties of the chemotype, such as, for example, up to 20 or 120 minutes for evaluation of prodrugs (e.g., $\mathrm{CL}_{\text {int }}>300 \mu \mathrm{l} / \mathrm{min}$ per milligram) or low-clearance compounds $\left(\mathrm{CL}_{\text {int }}<3 \mu \mathrm{l} / \mathrm{min}\right.$ per milligram), respectively. 

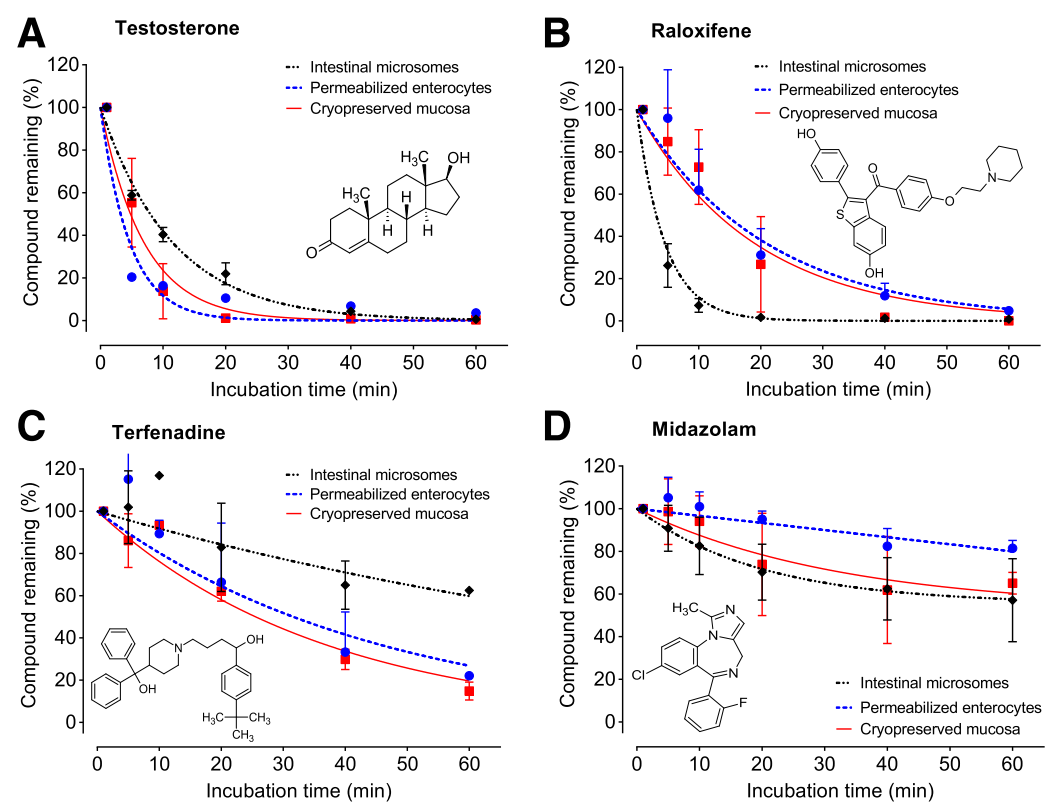

Fig. 2. An example of $C L_{\text {int }}$ measurements performed for compounds with low (A and B), medium (C and D), and high (E and F) observed human $f_{\mathrm{g}}$ values. Experiments were performed with human intestinal microsomes, permeabilized enterocytes, and cryopreserved mucosa. Presented data points are mean values \pm S.D. from at least three independent experiments. Observed human $f_{\mathrm{g}}$ values for the given compounds were (order from A to F) $0.05,0.05,0.40,0.55$, 0.94 , and 0.79 , respectively.
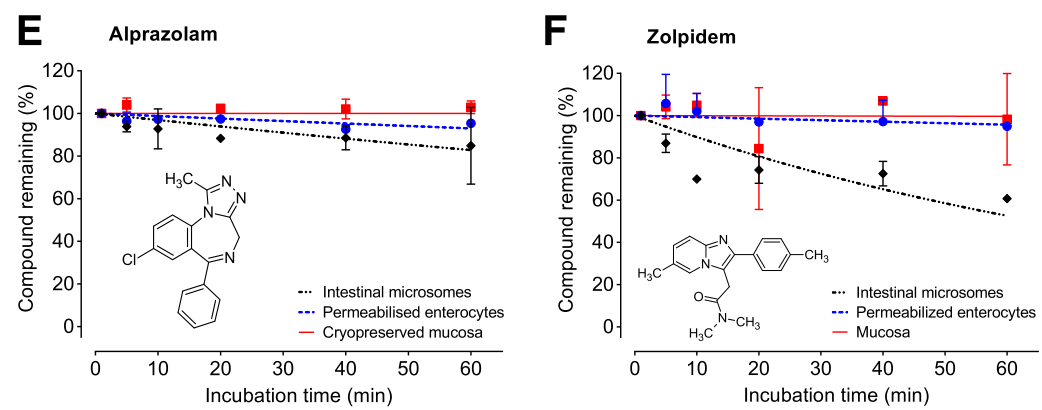

Biotransformation Pathways. We have analyzed our in vitro samples with QuanQual bioanalytical approach to simultaneously measure both parent compound disappearance and metabolite appearance. In contrast to compound disappearance data used to calculate $\mathrm{CL}_{\mathrm{int}}$, since metabolites authentic standards were not used, obtained metabolite formation data are predominantly qualitative. Four compounds were selected to exemplify the utility of this approach: midazolam, buspirone, raloxifene, and dabigatran etexilate. Example data for incubations with permeabilized enterocytes, together with corresponding biotransformation schemes, are presented in Fig. 3 (remaining results for intestinal microsomes and mucosa are presented in Supplemental Fig. 7).

Midazolam, a positive control for CYP3A4 metabolism used in all of our assays, showed formation of one prominent $+\mathrm{O}$ metabolite, likely corresponding to the $1^{\prime}-\mathrm{OH}$-midazolam, the most abundant oxidative metabolite of midazolam at low substrate concentrations (Gorski et al., 1994) (Fig. 3A). Several other metabolites of midazolam were observed at the lower level, including $+\mathrm{O}$ (likely a $4-\mathrm{OH}$ midazolam), $+\mathrm{O}-2 \mathrm{H}$, and $+2 \mathrm{O}$ metabolites (unpublished data). Buspirone, a dopaminergic anxiolytic and CYP3A4 substrate (Zhu et al., 2005), showed formation of five distinct $+\mathrm{O}$ metabolites in human enterocytes, consistent with previous reports (Gammans et al., 1986; Zhu et al., 2005) (Fig. 3B).

Raloxifene, a bis-phenolic estrogen receptor modulator, is known to be both glucoronidated (Kemp et al., 2002; Dalvie et al., 2008) and sulfated (Falany et al., 2006). Both types of metabolites were observed in our incubations with human enterocytes (Fig. 3C), showing that key conjugative biotransformation pathways are present. Raloxifene sulfation, catalyzed by cytosolic sulfotransferases, was observed in human enterocytes and mucosa but was predictably absent in human intestinal microsomes (Supplemental Fig. 7), highlighting the advantages of cellular models. Dabigatran etexilate is a double prodrug of anticoagulant dabigatran (Laizure et al., 2014) containing a cleavable carbamate and ester functionalities. Both hydrolysis products were detected in our intestinal incubations (Fig. 3D), with CES2-catalyzed hydrolysis of carbamate being significantly more prominent (Laizure et al., 2014). Several other oxidative metabolites of dabigatran were also observed (unpublished data).

Fraction Unbound in the Incubation. The $f_{\mathrm{u}, \text { inc }}$ values were measured by RED for all test compounds $(n=32)$ in permeabilized enterocytes and, for a subset of compounds, in intestinal microsomes $(n=15)$ and mucosa $(n=8)$ (Table 3). Direct comparison of obtained $f_{\text {u,inc }}$ values showed similar nonspecific binding across three models (Fig. 4A; Supplemental Table 1: one-way ANOVA showed insignificant differences among mean values). As an example, the $f_{\mathrm{u} \text {,inc }}$ values for midazolam were $0.74,0.74$, and 0.71 in permeabilized enterocytes, intestinal microsomes, and mucosa, respectively (Supplemental Table 1). Therefore, the average $f_{\mathrm{u} \text {,inc }}$ values from permeabilized enterocytes were considered to be representative for in vitro nonspecific binding and used for further predictions of human $f_{\mathrm{g}}$. Measured $f_{\mathrm{u} \text {,inc }}$ values also showed a statistically significant negative correlation with $\log \mathrm{D}$ values (Fig. 4B) (Pearson correlation $r=-0.64 ; 95 \% \mathrm{CI}:-0.8120$ to $-0.3732 ; P<0.0001)$. Although this correlation may be used for predictive purposes, similar to predictions of $f_{\mathrm{u} \text {,inc }}$ in human hepatocytes (Austin et al., 2005), the nonlinear regression with quadratic equation resulted in relatively poor outcome $\left(f_{u, i n c}=0.9370-0.07149 \cdot \log D-0.01580 \cdot \log D^{2}\right.$; $\left.r^{2}=0.46\right)$. Because the relatively short equilibration time of 4 hours was used for all measurements, readers should note that $f_{\mathrm{u} \text {,inc }}$ values obtained for moderately bound to highly bound compounds may be less 

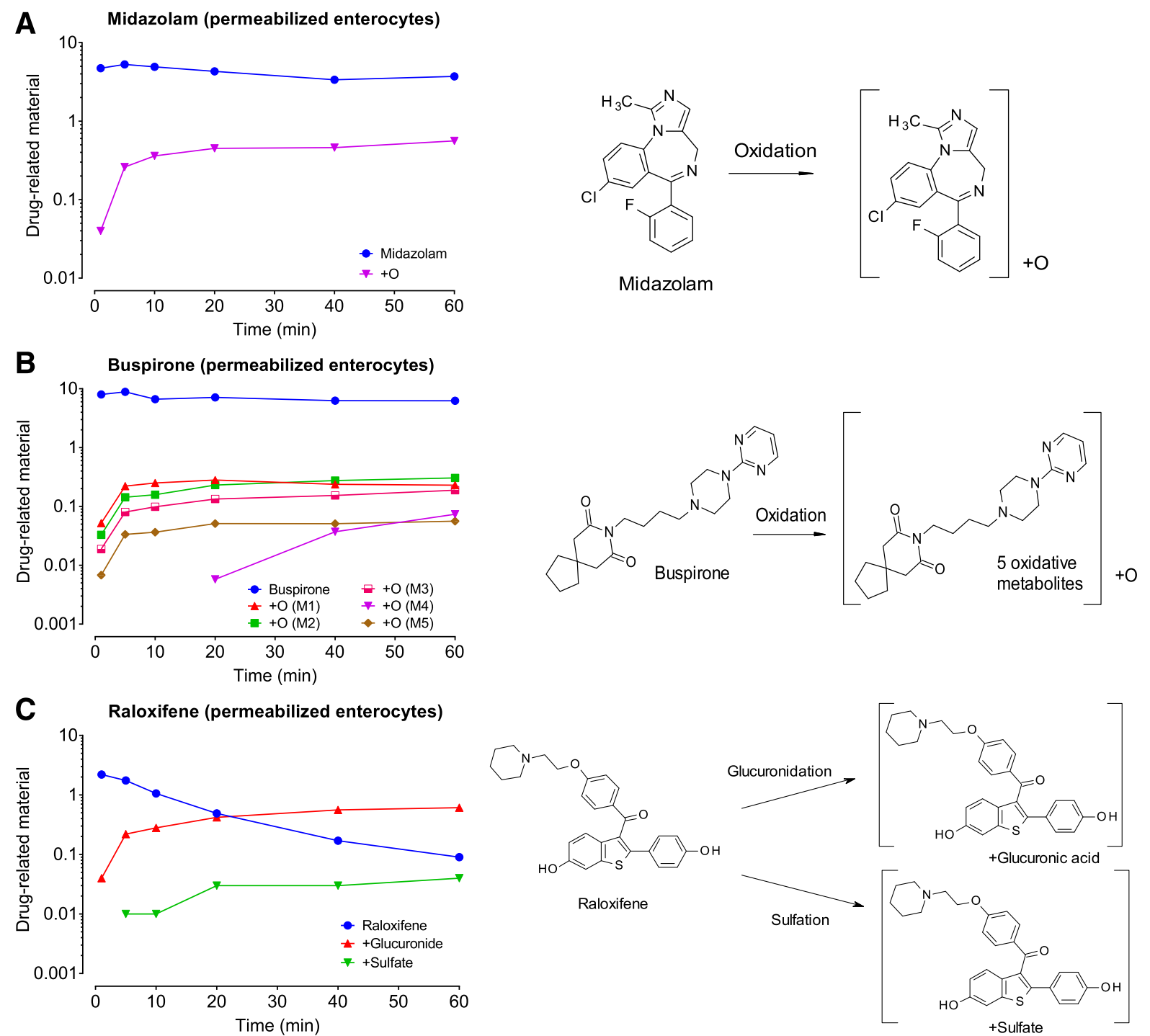

D Dabigatran etexilate (permeabilized enterocytes)

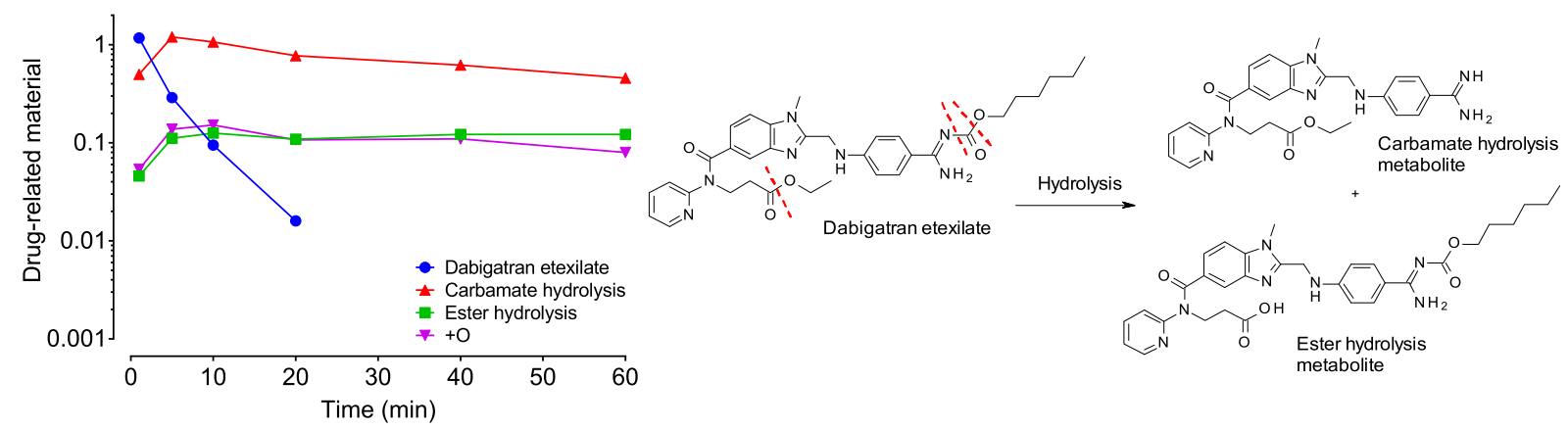

Fig. 3. Examples of biotransformation pathway analysis via the QuanQual approach (LC-HRMS). Although midazolam (A) and buspirone (B) predominantly form oxidative metabolites, raloxifene (C) undergoes conjugative metabolism (glucuronidation and sulfation), and dabigatran etexilate (D) is hydrolyzed (at the carbamate and amide functionalities). The $y$-axis units, drug-related material, represent LC-HRMS peak areas of either parent drug or corresponding drug metabolites. Because authentic standards were not used, presented data should be regarded as mainly qualitative.

accurate (e.g., terfenadine; $f_{\text {u,inc }}=0.05$ ) and should be ideally confirmed with longer equilibration time or orthogonal methods (Riccardi et al., 2015; Chen et al., 2019).

Early Predictions of Human $f_{\mathbf{g}}$ Values. Measured in vitro results $\left(\mathrm{CL}_{\text {int }}\right.$ and $\left.f_{\mathrm{u} \text {,inc }}\right)$ were supplemented with literature data (passive Caco-2 $\mathrm{A} \rightarrow \mathrm{B} P_{\mathrm{app}}, Q_{\mathrm{ent}}$, and $A_{\text {int }}$ ) and combined using a midazolam-calibrated $Q_{\text {gut }}$ model to predict human $f_{\mathrm{g}}$ values (see Data Analysis for details).
Predicted human $f_{\mathrm{g}}$ values were then compared with observed human $f_{\mathrm{g}}$ values from the literature (Fig. 5; Table 3). In general, all three in vitro models offered a similar predictivity of human $f_{\mathrm{g}}$ values, with GMFE values of 2.62, 2.81, and 2.81 for intestinal microsomes, permeabilized enterocytes, and intestinal mucosa, respectively (Table 4). If compounds are arbitrarily classified according to their observed $f_{\mathrm{g}}$ values, the best predictivity was achieved for the high $-f_{\mathrm{g}}$ compounds $\left(f_{\mathrm{g}}>0.70\right.$; GMFE 
TABLE 3

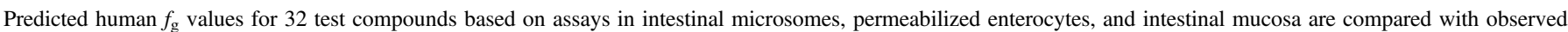
in vivo $f_{\mathrm{g}}$ values from the literature

See Table 4 for the accuracy and bias of human $f_{g}$ predictions. Values are presented as mean \pm S.E.

\begin{tabular}{|c|c|c|c|c|}
\hline \multirow[b]{2}{*}{ Compound } & \multirow[b]{2}{*}{$\begin{array}{l}\text { Observed } \\
f_{\mathrm{g}} \text { Value }\end{array}$} & \multicolumn{3}{|c|}{ Predicted Human $f_{\mathrm{g}}$ Values } \\
\hline & & Intestinal Microsomes & $\begin{array}{l}\text { Permeabilized } \\
\text { Enterocytes }\end{array}$ & Intestinal Mucosa \\
\hline Dabigatran etexilate & 0.05 & $0.20 \pm 0.01$ & $0.02 \pm 0.00$ & $0.04 \pm 0.00$ \\
\hline Raloxifene & 0.05 & $0.01 \pm 0.00$ & $0.03 \pm 0.00$ & $0.02 \pm 0.00$ \\
\hline Testosterone & 0.05 & $0.16 \pm 0.01$ & $0.03 \pm 0.00$ & $0.04 \pm 0.00$ \\
\hline Lovastatin & 0.07 & $0.05 \pm 0.01$ & $0.02 \pm 0.00$ & $0.02 \pm 0.00$ \\
\hline Nisoldipine & 0.11 & $0.04 \pm 0.00$ & $0.02 \pm 0.00$ & $0.02 \pm 0.00$ \\
\hline Buspirone & 0.18 & $0.79 \pm 0.10$ & $0.63 \pm 0.03$ & $0.57 \pm 0.03$ \\
\hline Rifabutin & 0.21 & $0.78 \pm 0.08$ & $0.28 \pm 0.01$ & $0.93 \pm 0.12$ \\
\hline Saquinavir & 0.28 & $0.01 \pm 0.00$ & $0.01 \pm 0.00$ & $0.01 \pm 0.00$ \\
\hline Simvastatin & 0.29 & $0.95 \pm 0.07$ & $0.46 \pm 0.04$ & $0.58 \pm 0.35$ \\
\hline Atorvastatin & 0.40 & $0.96 \pm 0.13$ & $0.72 \pm 0.06$ & $1.00 \pm 0.00$ \\
\hline Terfenadine & 0.40 & $0.01 \pm 0.00$ & $0.01 \pm 0.00$ & $0.01 \pm 0.00$ \\
\hline Felodipine & 0.42 & $0.02 \pm 0.00$ & $0.01 \pm 0.00$ & $0.01 \pm 0.00$ \\
\hline Cyclosporin & 0.48 & $0.01 \pm 0.00$ & $0.01 \pm 0.00$ & $0.01 \pm 0.00$ \\
\hline Midazolam & 0.55 & $0.55 \pm 0.03$ & $0.55 \pm 0.03$ & $0.55 \pm 0.04$ \\
\hline Cisapride & 0.57 & $0.82 \pm 0.10$ & $0.32 \pm 0.01$ & $0.34 \pm 0.03$ \\
\hline Ramipril & 0.59 & $0.68 \pm 0.00$ & $0.63 \pm 0.45$ & $0.85 \pm 0.00$ \\
\hline Verapamil & 0.60 & $0.82 \pm 0.08$ & $0.19 \pm 0.00$ & $0.13 \pm 0.01$ \\
\hline Sildenafil & 0.62 & $0.73 \pm 0.05$ & $0.84 \pm 0.05$ & $1.00 \pm 0.26$ \\
\hline Nifedipine & 0.71 & $0.86 \pm 0.10$ & $0.54 \pm 0.02$ & $0.92 \pm 0.04$ \\
\hline Diclofenac & 0.78 & $0.36 \pm 0.03$ & $0.56 \pm 0.02$ & $0.74 \pm 0.01$ \\
\hline Methadone & 0.78 & $0.99 \pm 0.00$ & $0.45 \pm 0.02$ & $0.87 \pm 0.03$ \\
\hline Zolpidem & 0.79 & $0.71 \pm 0.07$ & $0.80 \pm 0.05$ & $1.00 \pm 0.00$ \\
\hline Trazodone & 0.83 & $0.99 \pm 0.00$ & $0.68 \pm 0.04$ & $1.00 \pm 0.00$ \\
\hline Repaglinide & 0.89 & $0.83 \pm 0.13$ & $0.84 \pm 0.08$ & $1.00 \pm 0.05$ \\
\hline Quinidine & 0.91 & $0.99 \pm 0.00$ & $0.92 \pm 0.07$ & $1.00 \pm 0.00$ \\
\hline Indinavir & 0.93 & $0.63 \pm 0.09$ & $0.18 \pm 0.01$ & $0.99 \pm 0.09$ \\
\hline Alprazolam & 0.94 & $0.90 \pm 0.06$ & $0.87 \pm 0.05$ & $1.00 \pm 0.04$ \\
\hline Enalapril & 0.97 & $0.79 \pm 0.00$ & $0.73 \pm 0.36$ & $1.00 \pm 0.00$ \\
\hline Benzydamine & 0.98 & $0.97 \pm 0.00$ & $0.22 \pm 0.01$ & $0.41 \pm 0.02$ \\
\hline Carbazeran & $\mathrm{NA}^{a}$ & $0.91 \pm 0.04$ & $0.93 \pm 0.06$ & $0.99 \pm 0.00$ \\
\hline$S$-Mephenytoin & $\mathrm{NA}^{a}$ & $0.85 \pm 0.03$ & $0.93 \pm 0.06$ & $1.00 \pm 0.00$ \\
\hline Terbutaline & $\mathrm{NA}^{a}$ & $1.00 \pm 0.00$ & $1.00 \pm 0.00$ & $1.00 \pm 0.00$ \\
\hline
\end{tabular}

NA, data not available

${ }^{a}$ Insufficient amount of information in the literature to calculate human $f_{\mathrm{g}}$.

$1.23,1.53$, and 1.21 , respectively). However, the predictivity for low $\left(f_{\mathrm{g}}<0.30 ;\right.$ GMFE $4.21,3.26$, and 3.85 , respectively) and especially moderate $\left(0.30<f_{\mathrm{g}}<0.70 ;\right.$ GMFE $4.12,5.06$, and 5.76 , respectively $) f_{\mathrm{g}}$ compounds was significantly inferior (Table 4).

Predictions of human $f_{\mathrm{g}}$ can also be evaluated from the perspective of classification success (i.e., low, moderate, and high) and prediction bias (Table 4). Similar to GMFE, classification success analysis showed that only high $-f_{\mathrm{g}}$ compounds are acceptably classified based on predictions, especially based on intestinal microsomes $(81.8 \%)$ and intestinal mucosa $(90.9 \%)$. The classification success of high- $f_{\mathrm{g}}$ compounds based on permeabilized enterocytes was lower $(45.5 \%)$, with many compounds classified into moderate- $f_{\mathrm{g}}$ group instead of high, however. This phenomenon is related to the observed $f_{\mathrm{g}}$ underprediction bias in permeabilized enterocytes (i.e., higher metabolism rate) (Table 4). The classification success was acceptable for the low- $f_{\mathrm{g}}$ compounds (average $66.7 \%$ ) but poor for moderate- $f_{\mathrm{g}}$ compounds (average $25.9 \%$ ) (Table 4 ). Prediction bias was generally not pronounced (Table 4), except in the above mentioned permeabilized enterocytes and high- $f_{\mathrm{g}}$ compounds.

The $f_{\mathrm{g}}$ predictions for certain compounds varied significantly between different models (e.g., indinavir and rifabutin). Indinavir was significantly underpredicted in permeabilized enterocytes (predicted $f_{\mathrm{g}}$ of 0.16 vs. observed $f_{\mathrm{g}}$ of 0.91 ) but better predicted in intestinal microsomes and mucosa $\left(f_{\mathrm{g}}\right.$ of 0.56 and 0.84 , respectively). Conversely, rifabutin was well predicted in permeabilized enterocytes (predicted $f_{\mathrm{g}}$ of $0.40 \mathrm{vs}$. observed $f_{\mathrm{g}}$ of 0.21 ) but was poorly predicted in intestinal microsomes and mucosa $\left(f_{\mathrm{g}}\right.$ of 0.79 and 0.93 , respectively).
Saturation and Enzyme Kinetics Experiments. Because all $\mathrm{CL}_{\text {int }}$ measurements were initially performed at $1 \mu \mathrm{M}$ of the test substrate, which is typical of drug discovery screening assays, further experiments were performed to evaluate the effects of enzyme saturation on metabolic rates and accuracy of human $f_{\mathrm{g}}$ predictions. These experiments were performed with permeabilized enterocytes and two test substrates: indinavir $\left(f_{\mathrm{g}}\right.$ underpredicted by $\left.72 \%\right)$ and raloxifene $\left(f_{\mathrm{g}}\right.$ underpredicted by $2 \%$ ). Two example compounds were selected based on accuracy of initial $f_{\mathrm{g}}$ predictions and diversity of biotransformation pathways (oxidative and conjugative metabolism for indinavir and raloxifene, respectively). In addition to $\mathrm{CL}_{\text {int }}$, metabolite formation data were used to estimate the apparent $K_{\mathrm{m}}$ values. Results show that indinavir exhibits pronounced saturations of its metabolism (Fig. 6A), with apparent $\mathrm{CL}_{\text {int }}$ decreasing 10-fold within the concentration range of $0.1-5 \mu \mathrm{M}$ (from 32.24 to $3.35 \mu \mathrm{l} / \mathrm{min}$ per $10^{6}$ cells). The apparent $\mathrm{CL}_{\text {int }}$ at higher concentrations of indinavir $(>5 \mu \mathrm{M})$ was undetectable $(<1 \mu \mathrm{l} / \mathrm{min}$ per $10^{6}$ cells). The apparent $K_{\mathrm{m}}$ value of indinavir oxidative metabolism, based on the sum of all observed metabolites, was 1.24 $\mu \mathrm{M}$ (Fig. 6B). In contrast to indinavir, metabolism of raloxifene showed less saturation, with relatively modest 2-fold decrease of the observed $\mathrm{CL}_{\text {int }}$ at $0.1-5 \mu \mathrm{M}$ (from 234 to $125 \mu \mathrm{l} / \mathrm{min}$ per $10^{6}$ cells; Fig. $6 \mathrm{C}$ ) and apparent $K_{\mathrm{m}}$ value of conjugative metabolism of $8.4 \mu \mathrm{M}$ (glucuronidation and sulfation) (Fig. 6D). Further increase of raloxifene concentration resulted in additional decrease of observed $\mathrm{CL}_{\text {int }}$, with lowest value of $3.24 \mu \mathrm{l} / \mathrm{min}$ per $10^{6}$ cells at $100 \mu \mathrm{M}$ of the substrate (Fig. 6C). The $K_{\mathrm{m}}$ values determined with an alternative method directly from the substrate 


\section{A \\ Correlation of measured $f_{\mathrm{u} \text {,inc }}$ between three intestinal models}

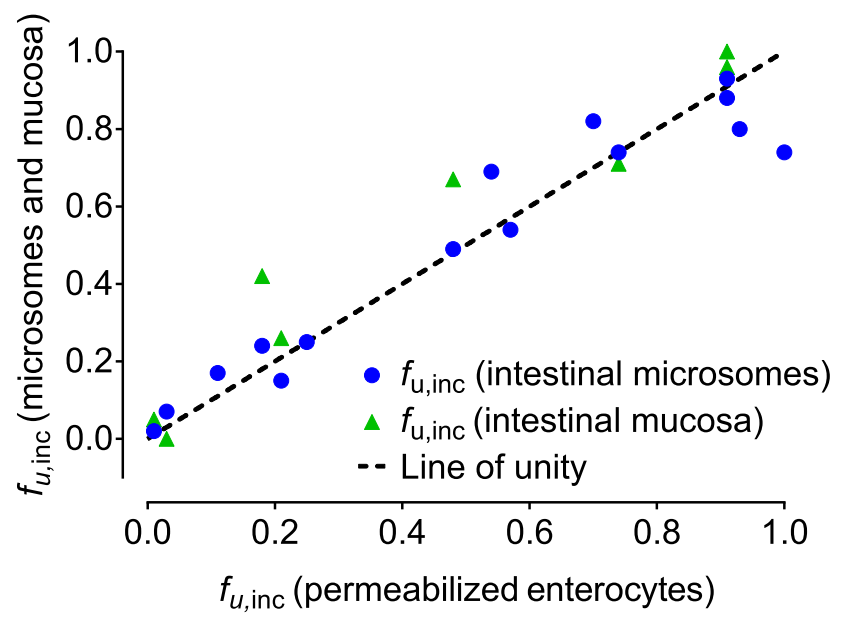

\section{B Correlation of measured $f_{\mathrm{u}, \mathrm{inc}}$ with $\log \mathrm{D}(\mathrm{pH} 7.4)$}

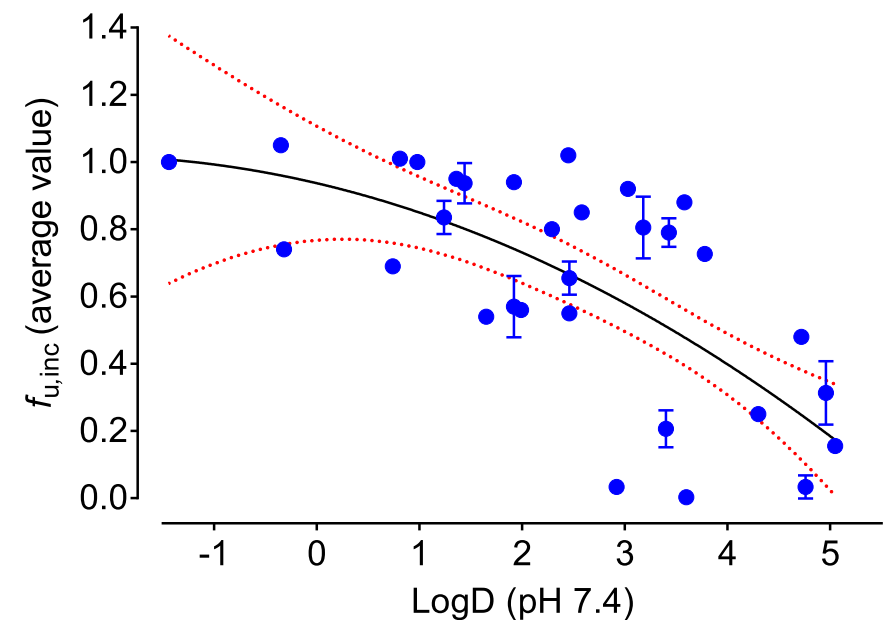

Fig. 4. A comparison of observed $f_{\mathrm{u} \text {,inc }}$ values for 32 test compounds in human intestinal microsomes, permeabilized enterocytes, and intestinal mucosa (A). A correlation of average $f_{\mathrm{u} \text {,inc }}$ with lipophilicity $(\log \mathrm{D}$ at $\mathrm{pH} 7.4)(\mathrm{B})$. The $f_{\mathrm{u} \text {,inc }}$ values were measured at $10 \mu \mathrm{M}$ of the substrate after 4 hours of equilibrium with the RED device.

disappearance data (Obach and Reed-Hagen, 2002) were comparable to metabolite formation approach [indinavir $K_{\mathrm{m}}=0.63 \pm 0.11 \mu \mathrm{M}$ (mean \pm S.E.), $95 \%$ CI 0.36-1.12 $\mu \mathrm{M}$; raloxifene $4.73 \pm 0.67 \mu \mathrm{M}$ (mean \pm S.E.), 95\% CI 3.31-6.69 $\mu \mathrm{M}]$. Taken together, these results suggest that in-depth evaluations of intestinal drug metabolism should take into account luminal concentrations of the drug rather than nominal concentrations of $1 \mu \mathrm{M}$. The challenge is, however, that human luminal drug concentrations are poorly understood at drug discovery stage, at least prior to knowledge of thermodynamic (biorelevant) solubilities and predictions of human efficacious dose. Therefore, assays at $1 \mu \mathrm{M}$ of substrate are still valuable in discovery settings, especially considering that hepatocyte incubations and/or enzyme phenotyping assays are also often performed at these concentrations.

\section{Discussion}

Early predictions of human drug disposition from in vitro data are important in both qualitative and quantitative drug discovery, especially for drug design, safety evaluation, and timely progression of successful molecules. Although intestinal drug metabolism may have a significant impact on drug disposition, it is often studied in either intestinal microsomes (Peters et al., 2016; Hatley et al., 2017a), a model that lacks many drug-metabolizing enzymes, or tissue slices (de Graaf et al., 2010; Li et al., 2016) and organotypic cultures (Almeqdadi et al., 2019) that are rarely available in drug discovery settings (Fig. 1). To address the existing gap, we have compared intestinal microsomes with two emerging models of the human intestine: permeabilized enterocytes and cryopreserved mucosa.

Evaluation with a set of 32 test compounds (Table 1) showed that common CYP- and UGT-mediated metabolism is well captured by all three models (Fig. 2; Table 2). On average, however, enterocytes and mucosa exhibited a 2-fold higher $\mathrm{CL}_{\text {int }}$ for CYP-mediated reactions, enabling higher sensitivity for the low-clearance compounds. The reasons behind higher CYP-mediated metabolism in enterocytes and mucosa are unclear at this point but may include higher CYP expression in tested batches, better preservation of enzymatic activities, partially different gut regions, or combination of all. Further studies will be needed to address these points, especially prior to broader adoption of these models in drug discovery. In addition, CYP activities compared with intestinal microsomes, enterocytes, and mucosa showed higher rates of CES2-catalyzed dabigatran etexilate hydrolysis (Laizure et al., 2014), FMO-catalyzed benzydamine oxidation, and sulfation of raloxifene and testosterone (Falany et al., 2006), which are indicative of a generally broader application scope. Permeabilized enterocytes may have the additional benefits of enabling assays with poorly permeable or cytotoxic compounds as well as enabling enzyme phenotyping by differential application of cosubstrates (Li et al., 2018b). Metabolic clearance of angiotensin-converting enzyme inhibitors ramipril and enalapril, which are predominantly hydrolyzed in the human liver (MacFadyen et al., 1993; Levitt and Schoemaker, 2006), was low in all tested in vitro intestinal systems. Similarly, AO-mediated oxidation of carbazeran was low in all systems, consistent with reported low expression level of this enzyme in the intestine (Moriwaki et al., 2001). Some of the differences in observed $\mathrm{CL}_{\text {int }}$ values may be attributed to partially different regions of the human gut from which tested models were prepared (see Material and Methods for details). Although human intestinal microsomes and permeabilized enterocytes were predominantly from duodenum and jejunum, respectively, cryopreserved mucosa was prepared from all three intestinal segments (duodenum, jejunum, and ileum).

In addition to $\mathrm{CL}_{\text {int }}$ measurements, QuanQual bioanalytical approach enabled direct observation of the metabolite formation and corresponding biotransformation pathways (Fig. 3). Based on examples of midazolam, buspirone, raloxifene, and dabigatran etexilate, this approach can identify key biotransformation pathways, including reactions of oxidation, hydrolysis, and conjugative metabolism. The qualitative profiles also provide important confirmation of the relevant metabolic capacity of these systems. Even if metabolite formation data based on LC-HRMS ionization response are qualitative, or semiquantitative at best (Blanz et al., 2017), this information is invaluable in drug discovery to guide compound design, identify challenges of in vitro-in vivo correlation (e.g., involvement of hydrolytic enzymes or UGTs), or directly compare and contrast intestinal metabolic profiles with liver and other relevant tissues. As an example, early identification of hydrolytic drug metabolism, which is often responsible for (unpredictable) high systemic clearance or prodrug conversion (Nishimuta et al., 2014; Di, 2019), is very important for drug design in lead generation and optimization before significant resources are invested in selected chemotype. Advances in mass spectrometry, especially nanospray techniques (Valaskovic et al., 2006; Schadt et al., 2011) and computational 


\section{Human intestinal microsomes}

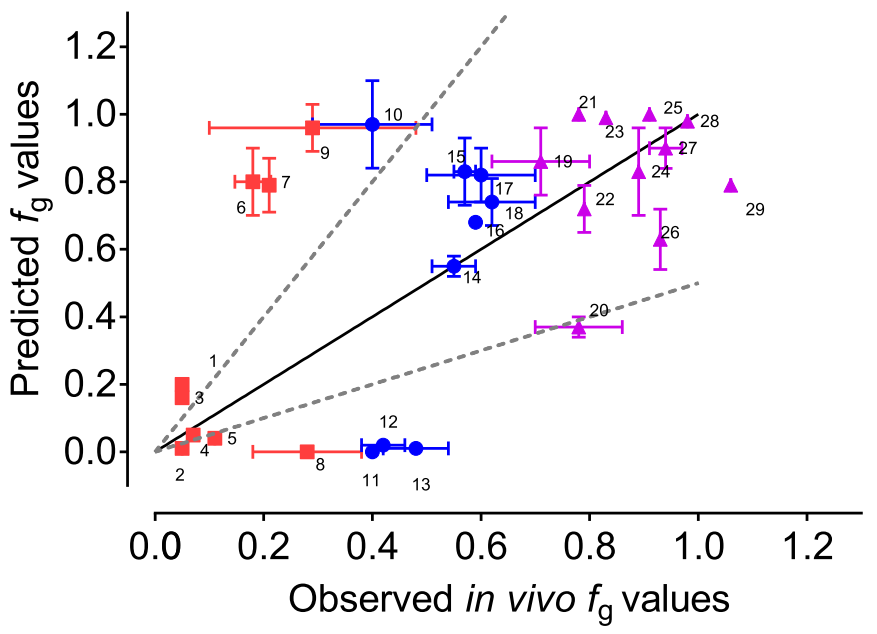

\section{B Human permeabilized enterocytes}
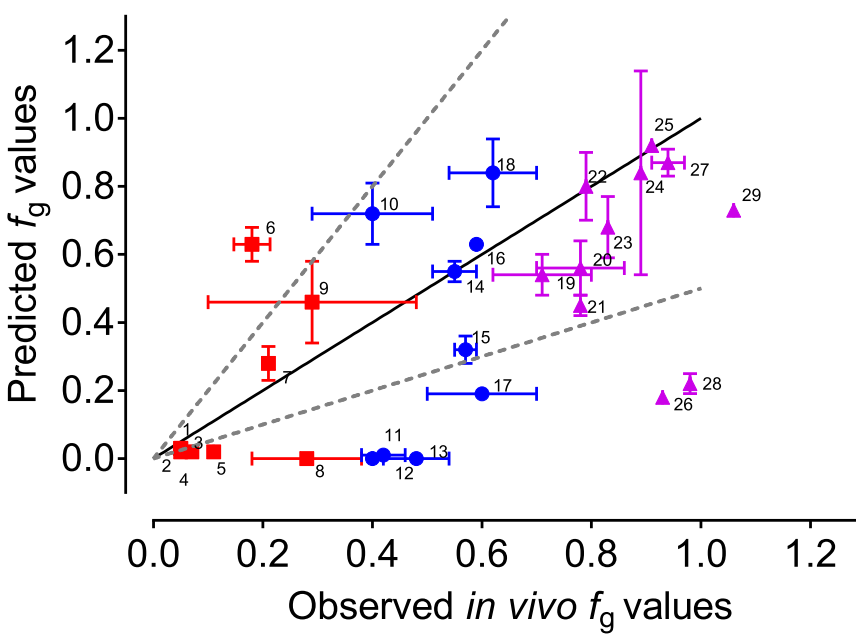

C

\section{Human cryopreserved intestinal mucosa}

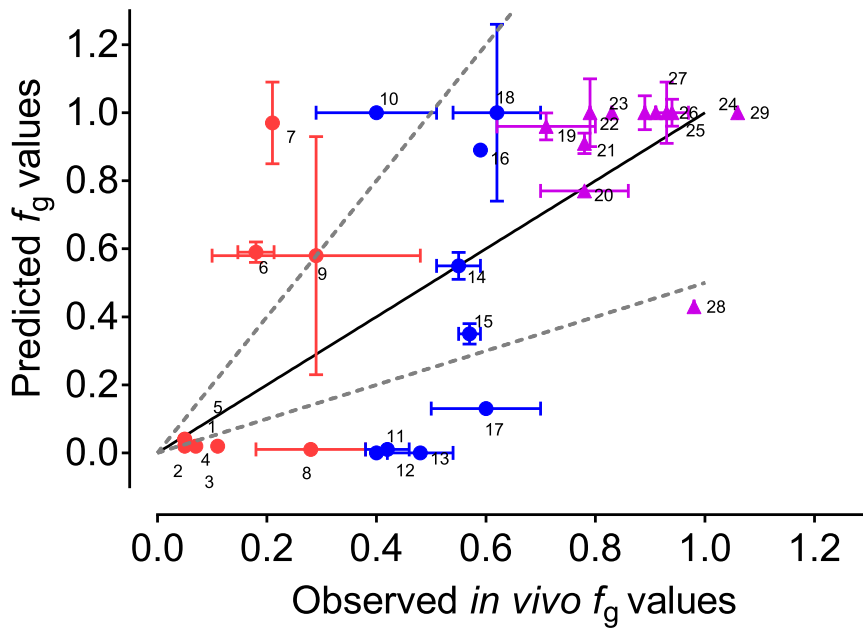

Fig. 5. Correlations between observed (in vivo) $f_{\mathrm{g}}$ values and predicted $f_{\mathrm{g}}$ values based on experiments in human intestinal microsomes (A), permeabilized enterocytes (B), and intestinal mucosa (C). Predicted $f_{\mathrm{g}}$ values were calculated based on the midazolam-calibrated $Q_{\text {gut }}$ model. Compounds are colored by the range of observed human $f_{\mathrm{g}}$ values $(0.00-0.30 \mathrm{red} \mathbf{\mathrm { ma }}$; $0.31-0.69$ blue $\mathbf{0} ; 0.70-1.00$ prediction of ionization response (Cramer et al., 2017), may be able to overcome analytical challenges in the future and provide quantitative data on drug metabolites even without authentic standards.

As shown by the equilibrium dialysis experiments (Fig. 4A; Supplemental Table 1), the in vitro nonspecific binding was similar across the three models and significantly correlated with lipophilicity (Fig. 4B). Although such an approach was often historically used to estimate liver $f_{u \text {,inc }}$ (Austin et al., 2002; Kilford et al., 2008), the predictivity of intestinal $f_{\mathrm{u} \text {,inc }}$ from the simple $\log \mathrm{D}$ correlation was limited, however, and experimental determination is recommended for important compounds. The fraction unbound in the gut is essentially unknown at the drug discovery stage and, similar to previous studies (Gertz et al., 2010), we have assumed a value of 1.

To attempt an early prediction of human $f_{\mathrm{g}}$ values, experimentally measured values $\left(\mathrm{CL}_{\mathrm{int}}\right.$ and $\left.f_{\mathrm{u} \text {,inc }}\right)$ were combined with literature data (Caco-2 A $\rightarrow \mathrm{B} P_{\text {app }}, Q_{\text {ent }}$, and $A_{\text {int }}$ ) using a midazolam-calibrated $Q_{\text {gut }}$ model (Fig. 5; Table 3). Results showed that early predictions of human $f_{\mathrm{g}}$ values are feasible, especially for compounds with the high observed human $f_{\mathrm{g}}$ values (arbitrarily $f_{\mathrm{g}}>0.70$; GMFE was $1.23,1.53$, and 1.21 for microsomes, enterocytes, and mucosa, respectively) (Table 4). Because most design teams and discovery projects aim for metabolically stable compounds with high $f_{\mathrm{g}}$ values and good oral bioavailability, this may be a valuable approach to perform early chemotype evaluations. The predictivity of low and moderate human $f \mathrm{~g}$ values remained a challenge, however, with average GMFEs of 3.77 and 4.98, respectively (Table 4). Although prediction accuracy in the low-tomoderate $-f_{\mathrm{g}}$ range is inadequate for decision making, it is sufficient to identify compounds at high risk and/or potential prodrug candidates. Overall, the presented approach may be useful for early assessments of the chemotype, with human $f_{\mathrm{g}}$ values $>0.7$ being well predicted and $f_{\mathrm{g}}$ values $<0.7$ suggesting further evaluation. Based on relatively poor predictivity of low-to-moderate- $f_{\mathrm{g}}$ values, authors would recommend against automatic avoidance of flagged compounds, many of which may show acceptable bioavailability after further evaluation. Additional experiments may include enzyme saturation, active transport, biorelevant solubilities, and application of physiologically based pharmacokinetic (PBPK) approaches. These results are generally similar to previous applications of the $Q_{\text {gut }}$ model (Yang et al., 2007; Gertz et al., 2010; Karlsson et al., 2013).

Apart from variabilities arising from gut biology (e.g., drug transporters, transit times, and interindividual variability), clinical study design (e.g., number, age, sex, and disease state of subjects), and pharmacological treatment (e.g., drug dose and solubility), the accuracy of $f_{\mathrm{g}}$ predictions is also partially biased by the mathematical structure of the $Q_{\text {gut }}$ model in relation to $\mathrm{CL}_{\text {int }}$ (see Materials and Methods for equations). Namely, if $\mathrm{CL}_{\mathrm{int}}$ is high, the $f_{\mathrm{g}}$ is directly proportional to $Q_{\mathrm{gut}}$ and inversely proportional to $\mathrm{CL}_{\text {int }}\left(f_{g} \approx \frac{Q_{\text {gut }}}{C L_{\text {int }}}\right)$. Therefore, the errors of $Q_{\text {gut }}$ and $\mathrm{CL}_{\text {int }}$ will translate into corresponding errors of $f_{\mathrm{g}}$. Conversely, if $\mathrm{CL}_{\mathrm{int}}$ is low, $f_{\mathrm{g}}$ will be close to 1 , and errors of $\mathrm{CL}_{\text {int }}$ and $\mathrm{Q}_{\text {gut }}$ will play a lesser role $\left(f_{g} \approx 1\right)$. This bias of the $Q_{\text {gut }}$ model can explain our "apparent" better predictivity at the high observed $f_{\mathrm{g}}$ values.

To demonstrate the potential impact of enzyme saturation on $f_{\mathrm{g}}$ predictions, which are important at higher dose levels of well soluble compounds, example studies with indinavir and raloxifene were

purple $\square$ ). Values are represented as the mean values \pm S.E. Compounds were dabigatran etexilate 1 , raloxifene 2 , testosterone 3 , lovastatin 4 , nisoldipine 5 , buspirone 6 , rifabutin 7 , saquinavir 8 , simvastatin 9 , atorvastatin 10 , terfenadine 11 , felodipine 12 , cyclosporin 13 , midazolam 14 , cisapride 15 , ramipril 16 , verapamil 17 , sildenafil 18, nifedipine 19 , diclofenac 20 , methadone 21 , zolpidem 22 , trazodone 23 , repaglinide 24 , quinidine 25 , indinavir 26 , alprazolam 27 , benzydamine 28 , and enalapril 29 
TABLE 4

Accuracy (GMFE and classification success) and bias of human $f_{\mathrm{g}}$ predictions based on in vitro assays in three intestinal models

\begin{tabular}{|c|c|c|c|}
\hline & $\begin{array}{l}\text { Intestinal } \\
\text { Microsomes }\end{array}$ & $\begin{array}{l}\text { Permeabilized } \\
\text { Enterocytes }\end{array}$ & $\begin{array}{l}\text { Intestinal } \\
\text { Mucosa }\end{array}$ \\
\hline \multicolumn{4}{|l|}{ GMFE } \\
\hline Overall & 2.62 & 2.81 & 2.81 \\
\hline Low $\left(f_{\mathrm{g}} 0-0.3\right)$ & 4.21 & 3.26 & 3.85 \\
\hline $\begin{array}{l}\text { Moderate }\left(f_{\mathrm{g}}\right. \\
0.31-0.70)\end{array}$ & 4.12 & 5.06 & 5.76 \\
\hline High $\left(f_{\mathrm{g}} 0.71-1.0\right)$ & 1.23 & 1.53 & 1.21 \\
\hline \multicolumn{4}{|l|}{ Classification success } \\
\hline Overall & $58.6 \%$ & $51.7 \%$ & $62.1 \%$ \\
\hline Low $\left(f_{\mathrm{g}} 0-0.3\right)$ & $66.7 \%$ & $66.7 \%$ & $66.7 \%$ \\
\hline $\begin{array}{l}\text { Moderate }\left(f_{\mathrm{g}}\right. \\
0.31-0.70)\end{array}$ & $22.2 \%$ & $33.3 \%$ & $22.2 \%$ \\
\hline High $\left(f_{\mathrm{g}} 0.71-1.0\right)$ & $81.8 \%$ & $45.5 \%$ & $90.9 \%$ \\
\hline \multicolumn{4}{|l|}{ Overprediction bias } \\
\hline Overall & $50.0 \%$ & $28.6 \%$ & $50.0 \%$ \\
\hline Low $\left(f_{\mathrm{g}} 0-0.3\right)$ & $55.6 \%$ & $33.3 \%$ & $33.3 \%$ \\
\hline $\begin{array}{l}\text { Moderate }\left(f_{\mathrm{g}}\right. \\
0.31-0.70)\end{array}$ & $62.5 \%$ & $37.5 \%$ & $37.5 \%$ \\
\hline High $\left(f_{\mathrm{g}} 0.71-1.0\right)$ & $36.4 \%$ & $18.2 \%$ & $72.7 \%$ \\
\hline \multicolumn{4}{|l|}{ Underprediction bias } \\
\hline Overall & $50.0 \%$ & $71.4 \%$ & $50.0 \%$ \\
\hline Low $\left(f_{\mathrm{g}} 0-0.3\right)$ & $44.4 \%$ & $66.7 \%$ & $66.7 \%$ \\
\hline $\begin{array}{l}\text { Moderate }\left(f_{\mathrm{g}}\right. \\
0.31-0.70)\end{array}$ & $37.5 \%$ & $62.5 \%$ & $62.5 \%$ \\
\hline $\operatorname{High}\left(f_{\mathrm{g}} 0.71-1.0\right)$ & $63.6 \%$ & $81.8 \%$ & $27.3 \%$ \\
\hline
\end{tabular}

performed in permeabilized enterocytes (Fig. 6). Our results confirmed that indinavir metabolism by CYP3A4/5 is highly saturable (Koudriakova et al., 1998), leading to underprediction or overprediction of the intestinal metabolism depending on the concentrations achieved in the intestinal lumen. In addition to demonstrated saturation effects, the predictions of indinavir $f_{\mathrm{g}}$ (observed value of 0.93 ) were modeldependent: $0.63,0.18$, and 0.99 in intestinal microsomes, enterocytes, and mucosa, respectively. The saturation of raloxifene metabolism was less pronounced, however, indicating less uncertainty by performing initial assays at $1 \mu \mathrm{M}$ of the substrate. The $K_{\mathrm{m}}$ values determined based on substrate disappearance and metabolite formation were comparable (within 2-fold), providing both possibilities to drug discovery teams.

Because several efflux and uptake transporters are found in the small intestine (Muller et al., 2017; Oswald, 2019; Couto et al., 2020), drug transport may also impact observed human $f_{\mathrm{g}}$ values (Shugarts and Benet, 2009; Yau et al., 2017). For example, our test compounds indinavir and atorvastatin are known substrates for P-gp and breast cancer resistance protein (Estudante et al., 2013), respectively, efflux transporters that may lower the effective permeability and enterocytic intracellular concentrations. Conversely, atorvastatin, enalapril, and verapamil are substrates for organic anion-transporting polypeptides, peptide transporter 1, and organic cation transporters, respectively (Estudante et al., 2013), uptake transporters that may increase effective permeability and facilitate interactions with drug-metabolizing enzymes. In vitro, passive permeability and active transport are not rate-limiting in intestinal microsomes and permeabilized enterocytes, models that lack any significant barrier function, but may play a role in intestinal mucosa because of the presence of intact plasma membrane and transporters yet to be fully evaluated (Li et al., 2018a). Indeed, models like intestinal mucosa may be used in the future to evaluate the interplay between drug metabolism and transport; for example, in the case of dabigatran etexilate, both CES (Bernier et al., 2019) and P-gp (Laizure et al., 2014) play significant roles. This application, however, will require further investigation, both with respect to expression and function of intestinal transporters in these models of human gut.

Considering the relatively low predictivity of the human $f_{\mathrm{g}}$ based on the $Q_{\text {gut }}$ model (Table 4) independent from the applied in vitro model of the human gut, one may wonder whether static gut models will be useful in the future of drug discovery. The $Q_{\text {gut }}$ model is based on simple assumptions: It represents the gut as a single homogenous compartment with uniform permeability, blood flow, and distribution of enzymes while not considering the role of transporters and pharmacological
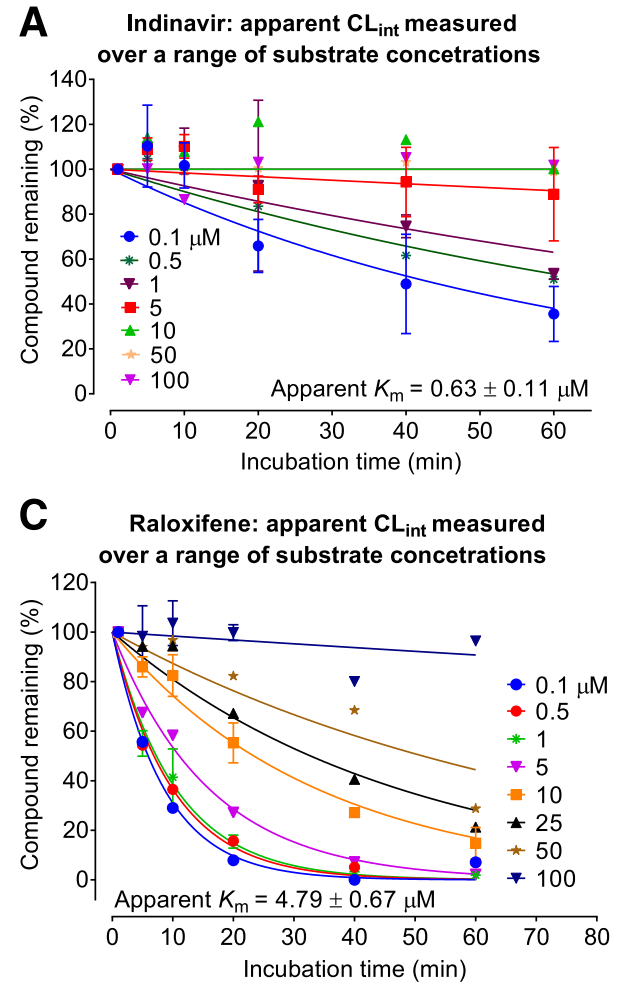

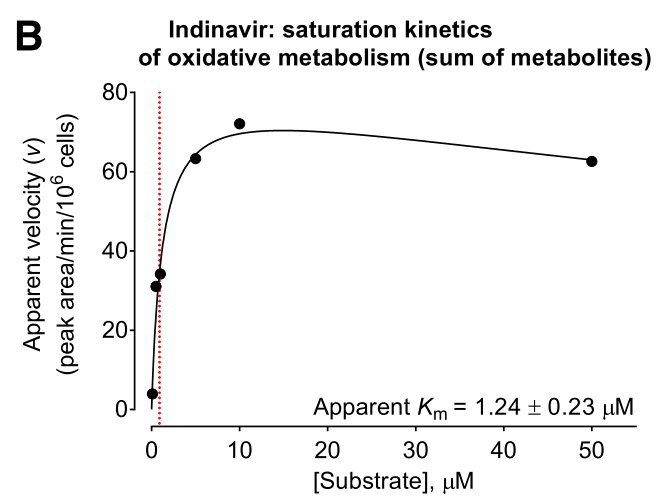

D

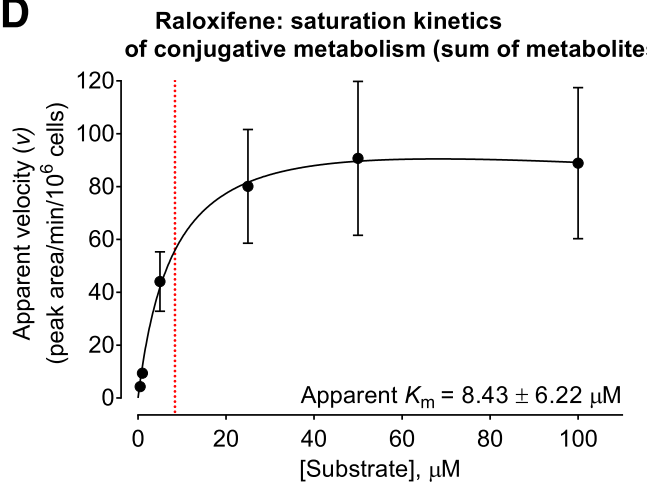

Fig. 6. Enzyme saturation experiments performed in permeabilized enterocytes to assess enzyme saturation as a reason for underpredictions and overpredictions of the human $f_{\mathrm{g}}$. (A and C) show substrate depletion of indinavir and raloxifene at increasing concentrations of the compound. (B and $\mathrm{D})$ show the rate of metabolite formation of indinavir and raloxifene with increasing substrate concentrations (sum of all observed metabolites used). The apparent $K_{\mathrm{M}}$ values of indinavir oxidation and raloxifene conjugation were calculated from both substrate disappearance $(0.63$ and $4.73 \mu \mathrm{M}$, respectively) and metabolite formation data (1.24 and $8.43 \mu \mathrm{M}$, respectively). Because drug metabolites were not quantified with authentic standards, obtained $V_{\max }$ values are qualitative and for relative comparisons only. 
treatment (e.g., drug dose and solubility). The future of drug discovery, therefore, will likely require PBPK models that can account for additional disposition mechanisms, including biorelevant solubilities, enterocytic concentrations, saturation of metabolism, and active transport. Indeed, an early application of intestinal PBPK model improved $f_{\mathrm{g}}$ predictions for indinavir, saquinavir, and terfenadine over the $Q_{\text {gut }}$ model (Gertz et al., 2011). Similarly, advanced dissolution absorption and metabolism (Darwich et al., 2010) and advanced compartmental and transit models (Gobeau et al., 2016) showed promise in drug discovery applications. The usefulness of intestinal PBPK models in drug discovery will be advanced with refined understanding of the gut physiology, especially with abundance of relevant enzymes and transporters (Couto et al., 2020) and computational approaches (i.e., machine learning/artificial intelligence) to predict compound properties based on the chemical structure alone-for example, biorelevant solubilities (Boobier et al., 2017) and transporter liabilities (Jain and Ecker, 2019).

In conclusion, we have evaluated three models of human intestine from the perspective of drug discovery. All three intestinal models showed reliable CYP and UGT enzymatic activities, but enterocytes and mucosa may offer advantages for low-clearance compounds and alternative biotransformation pathways (e.g., sulfation, hydrolases, and FMO oxidation). A number of knowledge gaps will need to be addressed in the future, including protein expression of drug-metabolizing enzymes and transporters and its overall predictivity of clinical outcomes. For the moment, applications of these models together with the QuanQual bioanalytical approach and midazolam-calibrated $Q_{\text {gut }}$ model enabled both qualitative (biotransformation pathways) and quantitative $\left(\mathrm{CL}_{\mathrm{int}}\right.$ and early $f_{\mathrm{g}}$ estimate) compound evaluation at the early stage, bringing together valuable information for drug design and discovery teams.

\section{Acknowledgments}

Authors would like to acknowledge University of Bath and Cogent Skills for support with student placement program. Vishal Ranglani is acknowledged for help with RED assay. Anna-Lena Ungell, Jamie Henshall, Peter Theil, and Emre Isin are greatly acknowledged for their support and encouragement. Lisa Jödicke is acknowledged for help with preparation of illustrations in Fig. 1. Albert Li and Behzad Emadi-Khiav from In Vitro ADMET Laboratories, LLC are acknowledged for help and support with human permeabilized enterocytes and cryopreserved mucosa.

\section{Authorship Contributions}

Participated in research design: Davies, Peramuhendige, Golding, Penney, Manevski.

Conducted experiments: Davies, Peramuhendige, Kotian, Shah, Manevski. Contributed new reagents or analytic tools: Peramuhendige, Kotian, Shah.

Performed data analysis: Davies, Peramuhendige, King, Penney, Shah, Manevski.

Wrote or contributed to the writing of the manuscript: Davies, Peramuhendige, King, Penney, Shah, Manevski.

\section{References}

Allen MJ, Powers ML, Gronowski KS, and Gronowski AM (2010) Human tissue ownership and use in research: what laboratorians and researchers should know. Clin Chem 56:1675-1682.

Almeqdadi M, Mana MD, Roper J, and Yilmaz OH (2019) Gut organoids: mini-tissues in culture to study intestinal physiology and disease. Am J Physiol Cell Physiol 317:C405-C419.

Alqahtani S, Bukhari I, Albassam A, and Alenazi M (2018) An update on the potential role of intestinal first-pass metabolism for the prediction of drug-drug interactions: the role of PBPK modeling. Expert Opin Drug Metab Toxicol 14:625-634.

Austin RP, Barton P, Cockroft SL, Wenlock MC, and Riley RJ (2002) The influence of nonspecific microsomal binding on apparent intrinsic clearance, and its prediction from physicochemical properties. Drug Metab Dispos 30:1497-1503.

Austin RP, Barton P, Mohmed S, and Riley RJ (2005) The binding of drugs to hepatocytes and it relationship to physicochemical properties. Drug Metab Dispos 33:419-425.

Baldock GA, Brodie RR, Chasseaud LF, Taylor T, Walmsley LM, and Catanese B (1991) Pharmacokinetics of benzydamine after intravenous, oral, and topical doses to human subjects. Biopharm Drug Dispos 12:481-492.

Benet LZ, Broccatelli F, and Oprea TI (2011) BDDCS applied to over 900 drugs. AAPS J 13: 519-547.
Bernier M, Lancrerot SL, Rocher F, Van-Obberghen EK, Olivier P, Lavrut T, Parassol-Girard N, and Drici MD (2019) Major bleeding events in octagenarians associated with drug interactions between dabigatran and P-gp inhibitors. $J$ Geriatr Cardiol 16:806-811.

Blanz J, Williams G, Dayer J, Délémonté T, Gertsch W, Ramstein P, Aichholz R, Trunzer M, and Pearson D (2017) Evaluation of relative MS response factors of drug metabolites for semi-quantitative assessment of chemical liabilities in drug discovery. J Mass Spectrom 52 : $210-217$

Blech S, Ebner T, Ludwig-Schwellinger E, Stangier J, and Roth W (2008) The metabolism and disposition of the oral direct thrombin inhibitor, dabigatran, in humans. Drug Metab Dispos 36 386-399.

Bohets H, Lavrijsen K, Hendrickx J, van Houdt J, van Genechten V, Verboven P, Meuldermans W, and Heykants J (2000) Identification of the cytochrome P450 enzymes involved in the metabolism of cisapride: in vitro studies of potential co-medication interactions. Br J Pharmacol 129: $1655-1667$.

Boobier S, Osbourn A, and Mitchell JBO (2017) Can human experts predict solubility better than computers? J Cheminform 9:63.

Burden N, Chapman K, Sewell F, and Robinson V (2015) Pioneering better science through the 3Rs: an introduction to the national centre for the replacement, refinement, and reduction of animals in research (NC3Rs). J Am Assoc Lab Anim Sci 54:198-208.

Buur A and Mørk N (1992) Metabolism of testosterone during in vitro transport across CACO-2 cell monolayers: evidence for beta-hydroxysteroid dehydrogenase activity in differentiated CACO-2 cells. Pharm Res 9:1290-1294.

Chen YC, Kenny JR, Wright M, Hop CECA, and Yan Z (2019) Improving confidence in the determination of free fraction for highly bound drugs using bidirectional equilibrium dialysis. J Pharm Sci 108:1296-1302.

Couto N, Al-Majdoub ZM, Gibson S, Davies PJ, Achour B, Harwood MD, Carlson G, Barber J, Rostami-Hodjegan A, and Warhurst G (2020) Quantitative proteomics of clinically relevant drug-metabolizing enzymes and drug transporters and their intercorrelations in the human small intestine. Drug Metab Dispos 48:245-254.

Cramer CJ, Johnson JL, and Kamel AM (2017) Prediction of mass spectral response factors from predicted chemometric data for druglike molecules. J Am Soc Mass Spectrom 28:278-285.

Cubitt HE, Houston JB, and Galetin A (2009) Relative importance of intestinal and hepatic glucuronidation-impact on the prediction of drug clearance. Pharm Res 26:1073-1083.

Dalvie D, Kang P, Zientek M, Xiang C, Zhou S, and Obach RS (2008) Effect of intestinal glucuronidation in limiting hepatic exposure and bioactivation of raloxifene in humans and rats. Chem Res Toxicol 21:2260-2271.

Darwich AS, Neuhoff S, Jamei M, and Rostami-Hodjegan A (2010) Interplay of metabolism and transport in determining oral drug absorption and gut wall metabolism: a simulation assessment using the "Advanced Dissolution, Absorption, Metabolism (ADAM)" model. Curr Drug Metab 11:716-729.

Davies B and Morris T (1993) Physiological parameters in laboratory animals and humans. Pharm Res 10:1093-1095.

Davies RO, Gomez HJ, Irvin JD, and Walker JF (1984) An overview of the clinical pharmacology of enalapril. Br J Clin Pharmacol 18 (Suppl 2):215S-229S.

de Graaf IA, Olinga P, de Jager MH, Merema MT, de Kanter R, van de Kerkhof EG, and Groothuis GM (2010) Preparation and incubation of precision-cut liver and intestinal slices for application in drug metabolism and toxicity studies. Nat Protoc 5:1540-1551.

Di L (2019) The impact of carboxylesterases in drug metabolism and pharmacokinetics. Curr Drug Metab 20:91-102.

Estudante M, Morais JG, Soveral G, and Benet LZ (2013) Intestinal drug transporters: an overview. Adv Drug Deliv Rev 65:1340-1356.

Falany JL, Pilloff DE, Leyh TS, and Falany CN (2006) Sulfation of raloxifene and 4 hydroxytamoxifen by human cytosolic sulfotransferases. Drug Metab Dispos 34:361-368.

Fisher MB, Campanale K, Ackermann BL, VandenBranden M, and Wrighton SA (2000) In vitro glucuronidation using human liver microsomes and the pore-forming peptide alamethicin. Drug Metab Dispos 28:560-566.

Galetin A, Burt H, Gibbons L, and Houston JB (2006) Prediction of time-dependent CYP3A4 drug-drug interactions: impact of enzyme degradation, parallel elimination pathways, and intestinal inhibition. Drug Metab Dispos 34:166-175.

Gammans RE, Mayol RF, and LaBudde JA (1986) Metabolism and disposition of buspirone. Am $J$ Med 80 (3B):41-51.

Gertz M, Davis JD, Harrison A, Houston JB, and Galetin A (2008) Grapefruit juice-drug interaction studies as a method to assess the extent of intestinal availability: utility and limitations. Curr Drug Metab 9:785-795.

Gertz M, Harrison A, Houston JB, and Galetin A (2010) Prediction of human intestinal first-pass metabolism of 25 CYP3A substrates from in vitro clearance and permeability data. Drug Metab Dispos 38:1147-1158.

Gertz M, Houston JB, and Galetin A (2011) Physiologically based pharmacokinetic modeling of intestinal first-pass metabolism of CYP3A substrates with high intestinal extraction. Drug Metab Dispos 39:1633-1642.

Gill KL, Houston JB, and Galetin A (2012) Characterization of in vitro glucuronidation clearance of a range of drugs in human kidney microsomes: comparison with liver and intestinal glucuronidation and impact of albumin. Drug Metab Dispos 40:825-835.

Gobeau N, Stringer R, De Buck S, Tuntland T, and Faller B (2016) Evaluation of the GastroPlus ${ }^{\text {TM }}$ Advanced Compartmental and Transit (ACAT) model in early discovery. Pharm Res 33: 2126-2139.

Gorski JC, Hall SD, Jones DR, VandenBranden M, and Wrighton SA (1994) Regioselective biotransformation of midazolam by members of the human cytochrome P450 3A (CYP3A) subfamily. Biochem Pharmacol 47:1643-1653.

Granger DN, Richardson PDI, Kvietys PR, and Mortillaro NA (1980) Intestinal blood flow. Gastroenterology 78:837-863.

Guo Y, Li Z, Su W, Wang L, Zhu Y, and Qin J (2018) A biomimetic human gut-on-a-chip for modeling drug metabolism in intestine. Artif Organs 42:1196-1205.

Hall SD, Thummel KE, Watkins PB, Lown KS, Benet LZ, Paine MF, Mayo RR, Turgeon DK, Bailey DG, Fontana RJ, et al. (1999) Molecular and physical mechanisms of first-pass extraction. Drug Metab Dispos 27:161-166.

Hanley MJ, Cancalon P, Widmer WW, and Greenblatt DJ (2011) The effect of grapefruit juice on drug disposition. Expert Opin Drug Metab Toxicol 7:267-286.

Hatley OJ, Jones CR, Galetin A, and Rostami-Hodjegan A (2017a) Quantifying gut wall metabolism: methodology matters. Biopharm Drug Dispos 38:155-160. 
Hatley OJD, Jones CR, Galetin A, and Rostami-Hodjegan A (2017b) Optimization of intestinal microsomal preparation in the rat: a systematic approach to assess the influence of various methodologies on metabolic activity and scaling factors. Biopharm Drug Dispos 38:187-208.

Hengstler JG, Utesch D, Steinberg P, Platt KL, Diener B, Ringel M, Swales N, Fischer T, Biefang K, Gerl M, et al. (2000) Cryopreserved primary hepatocytes as a constantly available in vitro model for the evaluation of human and animal drug metabolism and enzyme induction. Drug Metab Rev 32:81-118.

Hinz B, Chevts J, Renner B, Wuttke H, Rau T, Schmidt A, Szelenyi I, Brune K, and Werner U (2005) Bioavailability of diclofenac potassium at low doses. Br J Clin Pharmacol 59:80-84.

Ho MD, Ring N, Amaral K, Doshi U, and Li AP (2017) Human enterocytes as an in vitro model for the evaluation of intestinal drug metabolism: characterization of drug-metabolizing enzyme activities of cryopreserved human enterocytes from twenty-four donors. Drug Metab Dispos 45:686-691.

Hochman JH, Pudvah N, Qiu J, Yamazaki M, Tang C, Lin JH, and Prueksaritanont T (2004) Interactions of human P-glycoprotein with simvastatin, simvastatin acid, and atorvastatin. Pharm Res 21:1686-1691.

Ishiguro N, Kishimoto W, Volz A, Ludwig-Schwellinger E, Ebner T, and Schaefer O (2014) Impact of endogenous esterase activity on in vitro p-glycoprotein profiling of dabigatran etexilate in Caco-2 monolayers. Drug Metab Dispos 42:250-256.

Jacobson L, Middleton B, Holmgren J, Eirefelt S, Fröjd M, Blomgren A, and Gustavsson L (2007) An optimized automated assay for determination of metabolic stability using hepatocytes: assay validation, variance component analysis, and in vivo relevance. Assay Drug Dev Technol 5:403-415.

Jain S and Ecker GF (2019) In silico approaches to predict drug-transporter interaction profiles: data mining, model generation, and link to cholestasis. Methods Mol Biol 1981:383-396.

Jones CR, Hatley OJ, Ungell AL, Hilgendorf C, Peters SA, and Rostami-Hodjegan A (2016) Gut wall metabolism. Application of pre-clinical models for the prediction of human drug absorption and first-pass elimination. AAPS J 18:589-604.

Karlsson FH, Bouchene S, Hilgendorf C, Dolgos H, and Peters SA (2013) Utility of in vitro systems and preclinical data for the prediction of human intestinal first-pass metabolism during drug discovery and preclinical development. Drug Metab Dispos 41:2033-2046.

Kaye B, Offerman JL, Reid JL, Elliott HL, and Hillis WS (1984) A species difference in the presystemic metabolism of carbazeran in dog and man. Xenobiotica 14:935-945.

Kemp DC, Fan PW, and Stevens JC (2002) Characterization of raloxifene glucuronidation in vitro: contribution of intestinal metabolism to presystemic clearance. Drug Metab Dispos 30:694-700.

Kilford PJ, Gertz M, Houston JB, and Galetin A (2008) Hepatocellular binding of drugs: correction for unbound fraction in hepatocyte incubations using microsomal binding or drug lipophilicity data. Drug Metab Dispos 36:1194-1197.

Kosaka K, Sakai N, Endo Y, Fukuhara Y, Tsuda-Tsukimoto M, Ohtsuka T, Kino I, Tanimoto T, Takeba N, Takahashi M, et al. (2011) Impact of intestinal glucuronidation on the pharmacokinetics of raloxifene. Drug Metab Dispos 39:1495-1502.

Koudriakova T, Iatsimirskaia E, Utkin I, Gangl E, Vouros P, Storozhuk E, Orza D, Marinina J, and Gerber N (1998) Metabolism of the human immunodeficiency virus protease inhibitors indinavir and ritonavir by human intestinal microsomes and expressed cytochrome P4503A4/3A5: mechanismbased inactivation of cytochrome P4503A by ritonavir. Drug Metab Dispos 26:552-561.

Laizure SC, Parker RB, Herring VL, and Hu ZY (2014) Identification of carboxylesterasedependent dabigatran etexilate hydrolysis. Drug Metab Dispos 42:201-206.

Lang DH and Rettie AE (2000) In vitro evaluation of potential in vivo probes for human flavincontaining monooxygenase (FMO): metabolism of benzydamine and caffeine by FMO and P450 isoforms. Br J Clin Pharmacol 50:311-314.

Lee JB, Zgair A, Taha DA, Zang X, Kagan L, Kim TH, Kim MG, Yun HY, Fischer PM, and Gershkovich P (2017) Quantitative analysis of lab-to-lab variability in Caco-2 permeability assays. Eur J Pharm Biopharm 114:38-42.

Levitt DG and Schoemaker RC (2006) Human physiologically based pharmacokinetic model for ACE inhibitors: ramipril and ramiprilat. BMC Clin Pharmacol 6:1.

Li AP (2007) Human hepatocytes: isolation, cryopreservation and applications in drug development. Chem Biol Interact 168:16-29.

Li AP, Alam N, Amaral K, Ho MD, Loretz C, Mitchell W, and Yang Q (2018a) Cryopreserved human intestinal mucosal epithelium: a novel in vitro experimental system for the evaluation of enteric drug metabolism, cytochrome P450 induction, and enterotoxicity. Drug Metab Dispos 46:1562-1571.

Li AP, Amaral K, and Ho MD (2018b) A novel in vitro experimental system for the evaluation of enteric drug metabolism: cofactor-supplemented permeabilized cryopreserved human enterocytes (MetMax ${ }^{\mathrm{TM}}$ cryopreserved human enterocytes). Drug Metab Lett 12:132-137.

Li AP, Ho MD, Amaral K, and Loretz C (2018c) A novel in vitro experimental system for the evaluation of drug metabolism: cofactor-supplemented permeabilized cryopreserved human hepatocytes (MetMax cryopreserved human hepatocytes). Drug Metab Dispos 46:1608-1616.

Li M, de Graaf IA, and Groothuis GM (2016) Precision-cut intestinal slices: alternative model for drug transport, metabolism, and toxicology research. Expert Opin Drug Metab Toxicol 12:175-190.

Lu W, Rettenmeier E, Paszek M, Yueh MF, Tukey RH, Trottier J, Barbier O, and Chen S (2017) Crypt organoid culture as an in vitro model in drug metabolism and cytotoxicity studies. Drug Metab Dispos 45:748-754.

MacFadyen RJ, Meredith PA, and Elliott HL (1993) Enalapril clinical pharmacokinetics and pharmacokinetic-pharmacodynamic relationships. An overview. Clin Pharmacokinet 25:274-282.

Maresca M, Pinton P, Ajandouz EH, Menard S, Ferrier L, and Oswald IP (2018) Overview and comparison of intestinal organotypic models, intestinal cells, and intestinal explants used for toxicity studies. Curr Top Microbiol Immunol DOI: 10.1007/82_2018_142 [published ahead of print].

Mizuma T (2009) Intestinal glucuronidation metabolism may have a greater impact on oral bioavailability than hepatic glucuronidation metabolism in humans: a study with raloxifene, substrate for UGT1A1, 1A8, 1A9, and 1A10. Int J Pharm 378:140-141.

Mohri K and Uesawa Y (2001) Enzymatic activities in the microsomes prepared from rat small intestinal epithelial cells by differential procedures. Pharm Res 18:1232-1236.

Moriwaki Y, Yamamoto T, Takahashi S, Tsutsumi Z, and Hada T (2001) Widespread cellular distribution of aldehyde oxidase in human tissues found by immunohistochemistry staining. Histol Histopathol 16:745-753.

Müller J, Keiser M, Drozdzik M, and Oswald S (2017) Expression, regulation and function of intestinal drug transporters: an update. Biol Chem 398:175-192.

Nishimuta H, Houston JB, and Galetin A (2014) Hepatic, intestinal, renal, and plasma hydrolysis of prodrugs in human, cynomolgus monkey, dog, and rat: implications for in vitro-in vivo extrapolation of clearance of prodrugs. Drug Metab Dispos 42:1522-1531.

Nishimuta H, Sato K, Yabuki M, and Komuro S (2011) Prediction of the intestinal first-pass metabolism of CYP3A and UGT substrates in humans from in vitro data. Drug Metab Phar macokinet 26:592-601.
Obach RS and Reed-Hagen AE (2002) Measurement of Michaelis constants for cytochrome P450mediated biotransformation reactions using a substrate depletion approach. Drug Metab Dispos 30:831-837.

Oswald S (2019) Organic Anion Transporting Polypeptide (OATP) transporter expression, localization and function in the human intestine. Pharmacol Ther 195:39-53.

Paine CJ (1996) Nursing home patients: can feeding tubes be withheld? J La State Med Soc 148.284. Paine MF, Shen DD, Kunze KL, Perkins JD, Marsh CL, McVicar JP, Barr DM, Gillies BS, and Thummel KE (1996) First-pass metabolism of midazolam by the human intestine. Clin Pharmacol Ther 60:14-24.

Peters SA, Jones CR, Ungell AL, and Hatley OJ (2016) Predicting drug extraction in the human gut wall: assessing contributions from drug metabolizing enzymes and transporter proteins using preclinical models. Clin Pharmacokinet 55:673-696.

Polli JW, Wring SA, Humphreys JE, Huang L, Morgan JB, Webster LO, and Serabjit-Singh CS (2001) Rational use of in vitro P-glycoprotein assays in drug discovery. J Pharmacol Exp Ther 299:620-628.

Quignot N, Wiecek W, Amzal B, and Dorne JL (2019) The Yin-Yang of CYP3A4: a Bayesian meta-analysis to quantify inhibition and induction of CYP3A4 metabolism in humans and refine uncertainty factors for mixture risk assessment. Arch Toxicol 93:107-119.

Riccardi K, Cawley S, Yates PD, Chang C, Funk C, Niosi M, Lin J, and Di L (2015) Plasma protein binding of challenging compounds. J Pharm Sci 104:2627-2636.

Rostami-Hodjegan A and Tucker GT (2002) The effects of portal shunts on intestinal cytochrome P450 3A activity. Hepatology 35:1549-1550-1551.

Schadt S, Chen LZ, and Bischoff D (2011) Evaluation of relative LC/MS response of metabolites to parent drug in LC/nanospray ionization mass spectrometry: potential implications in MIST assessment. J Mass Spectrom 46:1281-1286.

Shugarts S and Benet LZ (2009) The role of transporters in the pharmacokinetics of orally administered drugs. Pharm Res 26:2039-2054.

Tachibana T, Kato M, Takano J, and Sugiyama Y (2010) Predicting drug-drug interactions involving the inhibition of intestinal CYP3A4 and P-glycoprotein. Curr Drug Metab 11:762-777.

Takahashi M, Uehara T, Nonaka M, Minagawa Y, Yamazaki R, Haba M, and Hosokawa M (2019) Synthesis and evaluation of haloperidol ester prodrugs metabolically activated by human carboxylesterase. Eur J Pharm Sci 132:125-131.

Täuber U, Schröder K, Düsterberg B, and Matthes H (1986) Absolute bioavailability of testosterone after oral administration of testosterone-undecanoate and testosterone. Eur J Drug Metab Pharmacokinet 11:145-149.

Taylor JR (1996) An Introduction to Error Analysis: The Study of Uncertainties in Physical Measurements, University Science Books, Sausalito, CA.

Valaskovic GA, Utley L, Lee MS, and Wu JT (2006) Ultra-low flow nanospray for the normalization of conventional liquid chromatography/mass spectrometry through equimolar response: standard-free quantitative estimation of metabolite levels in drug discovery. Rapid Commun Mass Spectrom 20:1087-1096.

van de Kerkhof EG, de Graaf IA, Ungell AL, and Groothuis GM (2008) Induction of metabolism and transport in human intestine: validation of precision-cut slices as a tool to study induction of drug metabolism in human intestine in vitro. Drug Metab Dispos 36:604-613.

van de Kerkhof EG, Ungell AL, Sjöberg AK, de Jager MH, Hilgendorf C, de Graaf IA, and Groothuis GM (2006) Innovative methods to study human intestinal drug metabolism in vitro: precision-cut slices compared with ussing chamber preparations. Drug Metab Dispos 34:1893-1902.

van Griensven JM, Schoemaker RC, Cohen AF, Luus HG, Seibert-Grafe M, and Röthig HJ (1995) Pharmacokinetics, pharmacodynamics and bioavailability of the ACE inhibitor ramipril. Eur J Clin Pharmacol 47:513-518.

Walsky RL, Bauman JN, Bourcier K, Giddens G, Lapham K, Negahban A, Ryder TF, Obach RS, Hyland R, and Goosen TC (2012) Optimized assays for human UDP-glucuronosyltransferase (UGT) activities: altered alamethicin concentration and utility to screen for UGT inhibitors. Drug Metab Dispos 40:1051-1065.

Wang D, Zou L, Jin Q, Hou J, Ge G, and Yang L (2018) Human carboxylesterases: a comprehensive review. Acta Pharm Sin B 8:699-712.

Wedlund PJ, Aslanian WS, Jacqz E, McAllister CB, Branch RA, and Wilkinson GR (1985) Phenotypic differences in mephenytoin pharmacokinetics in normal subjects. J Pharmacol Exp Ther 234:662-669.

Williams ET, Bacon JA, Bender DM, Lowinger JJ, Guo WK, Ehsani ME, Wang X, Wang H, Qian YW, Ruterbories KJ, et al. (2011) Characterization of the expression and activity of carboxylesterases 1 and 2 from the beagle dog, cynomolgus monkey, and human. Drug Metab Dispos 39:2305-2313.

Willis JV, Kendall MJ, Flinn RM, Thornhill DP, and Welling PG (1979) The pharmacokinetics of diclofenac sodium following intravenous and oral administration. Eur J Clin Pharmacol 16:405-410. Wong S, Doshi U, Vuong P, Liu N, Tay S, Le H, Kosaka M, Kenny JR, Li AP, and Yan Z (2018) Utility of pooled cryopreserved human enterocytes as an in vitro model for assessing intestinal clearance and drug-drug interactions. Drug Metab Lett 12:3-13.

Wu X, Whitfield LR, and Stewart BH (2000) Atorvastatin transport in the Caco-2 cell model: contributions of P-glycoprotein and the proton-monocarboxylic acid co-transporter. Pharm Res 17:209-215.

Yamada M, Inoue SI, Sugiyama D, Nishiya Y, Ishizuka T, Watanabe A, Watanabe K, Yamashita S, and Watanabe N (2020) Critical impact of drug-drug interactions via intestinal CYP3A in the risk assessment of weak perpetrators using physiologically based pharmacokinetic models. Drug Metab Dispos 48:288-296.

Yang J, Jamei M, Yeo KR, Tucker GT, and Rostami-Hodjegan A (2007) Prediction of intestinal first-pass drug metabolism. Curr Drug Metab 8:676-684.

Yang M, Ma J, Ruan J, Ye Y, Fu PP, and Lin G (2019) Intestinal and hepatic biotransformation of pyrrolizidine alkaloid N-oxides to toxic pyrrolizidine alkaloids. Arch Toxicol 93:2197-2209.

Yau E, Petersson C, Dolgos H, and Peters SA (2017) A comparative evaluation of models to predict human intestinal metabolism from nonclinical data. Biopharm Drug Dispos 38: $163-186$.

Zhu M, Zhao W, Jimenez H, Zhang D, Yeola S, Dai R, Vachharajani N, and Mitroka J (2005) Cytochrome P450 3A-mediated metabolism of buspirone in human liver microsomes. Drug Metab Dispos 33:500-507.

Address correspondence to: Dr. Nenad Manevski, UCB Celltech UK, 216 Bath Rd., Slough, SL1 3WE, United Kingdom. E-mail: Nenad.Manevski@gmail.com 